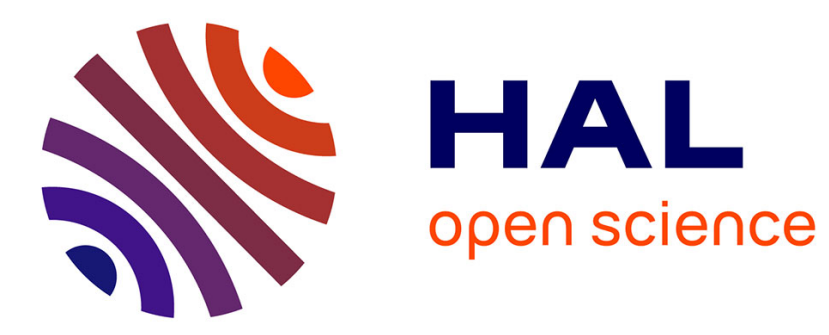

\title{
Numerical Study of Unsteady Turbulent Cavitating Flows
}

Eric Goncalvès da Silva

\section{To cite this version:}

Eric Goncalvès da Silva. Numerical Study of Unsteady Turbulent Cavitating Flows. European Journal of Mechanics - B/Fluids, 2011, 30, pp.26-40. 10.1016/j.euromechflu.2010.08.002 . hal-00530302

\section{HAL Id: hal-00530302 \\ https://hal.science/hal-00530302}

Submitted on 20 Dec 2012

HAL is a multi-disciplinary open access archive for the deposit and dissemination of scientific research documents, whether they are published or not. The documents may come from teaching and research institutions in France or abroad, or from public or private research centers.
L'archive ouverte pluridisciplinaire HAL, est destinée au dépôt et à la diffusion de documents scientifiques de niveau recherche, publiés ou non, émanant des établissements d'enseignement et de recherche français ou étrangers, des laboratoires publics ou privés. 


\title{
Numerical Study of Unsteady Turbulent Cavitating Flows
}

\author{
Eric Goncalvès * \\ LEGI-Grenoble INP, 1025 rue de la Piscine, 38400 St Martin d'Heres, France
}

\begin{abstract}
The simulation of cavitating flows is a challenging problem both in terms of modelling the physics and developing robust numerical methodologies. Such flows are characterized by important variations of the local Mach number, compressibility effects on turbulence and involve thermodynamic phase transition. To simulate these flows by applying homogeneous models and Reynolds averaged codes, the turbulence modelling plays a major role in the capture of unsteady behaviours. This paper presents a one-fluid compressible Reynolds-Averaged Navier-Stokes (RANS) solver with a simple equation of state (EOS) for the mixture. A special focus is devoted to the turbulence model influence. Unsteady numerical results are given for Venturi geometries and comparisons are made with experimental data.
\end{abstract}

Keywords: cavitation, homogeneous model, RANS simulations, turbulence model

\footnotetext{
*Corresponding author.

Email address: Eric.Goncalves@legi.grenoble-inp.fr (Eric Goncalvès )
} 


\section{Introduction}

The simulation and prediction of cavitating flows is of critical importance for the efficient design and performance of many engineering devices (turbomachinery, turbopumps in rocket propulsion systems, hydrofoils, fuel injectors, marine propellers, nozzles, underwater bodies, etc). In most cases, cavitation is an undesirable phenomenon, significantly degrading performance, resulting in reduced flow rates, lower pressure increases in pumps, load asymmetry, vibrations, noise and erosion. In most industrial applications, cavitating flows are turbulent and the dynamics of the interface formed involves complex interactions between the vapour and liquid phases. These interactions are not well understood in the closure region of cavities, where a distinct interface may not exist and where the flow is unsteady.

Various numerical models, within the framework of the continuum modelling method, have been developed to investigate cavitating flows. This method makes no attempt to track the liquid and vapour interface but treats the flow as two-phases with an averaged mixture density, which continuously varies between the liquid and vapour extremes. In its implementation, there are different approaches according to the assumptions made: equilibrium models versus non-equilibrium models, homogeneous models versus two-fluid models.

The two-fluid approach assumes that both phases co-exist at every point in the flow field and each phase is governed by its own set of conservation laws. These models can take into account the physical details occurring in the cavitation phenomenon such as mass exchange, thermal transfer and sur- 
face tension. However, transfer terms have to be known; such quantities are usually very difficult to obtain.

On the contrary, the one-fluid method treats the cavitating flows as a mixture of two fluids behaving as one. The governing equations are composed of three conservation laws written for the mixture. These models are based on the assumption of local kinematic equilibrium between phases (the local velocity is the same for both phases). With the assumption of local thermodynamic equilibrium (local temperature, pressure and free Gibbs enthalpy equality between phases), they constitute the homogeneous equilibrium models (HEM). These model cannot reproduce strong thermodynamic or kinetic non equilibrium effects but, because of its simplicity, it is often used for numerical simulations. An equation of state (EOS) is necessary to close the system. Different closure relations that link the pressure to the temperature and the density have been proposed: tabulated EOS $[1,2,3]$, a mixture entropy maximization procedure with a relaxed pressure law [4], barotropic mixture laws $[5,6,7,8,9,10,11,12]$, equilibrium EOS [13, 14].

When non-equilibrium effect becomes important, additional equations are needed for an accurate prediction. Hybrid or reduced models are intermediate models between one-fluid and two-fluid models, based on four or five equations. A four-equation model is obtained by adding a mass equation for the vapour or liquid density including a cavitation source term. This model is very popular in simulating cavitating flows [15, 16, 17, 18, 19, 20, 21, 22, 23]. The main difficulty is related to the formulation of the source term and the tunable parameters involved in the vaporization and condensation process (different formulations and sets of parameters are presented in [24]). 
In addition, the turbulence modelling plays a major role in the capture of unsteady behaviours. Cavitation sheets that appear on solid bodies are characterized by a closure region which always fluctuates with the existence of a re-entrant jet. This one is mainly composed of liquid which flows upstream along the solid surface. Moreover, due to the drastic decrease of the speed of sound in the mixture, large supersonic areas appear leading to compressibility effects on turbulence. These effects and interactions with two-phase structures are not yet well known and understood.

For usual applications, three-dimensional time-dependent computations obtained with large eddy simulations (LES) or direct simulations (DNS) are not yet tractable. The Reynolds decomposition is often used with an averaged statistical processing resulting in the RANS equations for the mean flow quantities. One fundamental problem with the RANS approach is that turbulence models are tuned by steady-state mean flow data. Moreover, the standard eddy-viscosity models based on the Boussinesq relation are known to over-product eddy-viscosity, which reduces the development of the re-entrant jet and two-phase structures shedding [25]. The limitation of the turbulent viscosity is a determinant point to capture realistic cavitation sheets. Different methods have been investigated to limit or to correct standard turbulence models. An arbitrary modification was proposed by Reboud to reduce the turbulent viscosity [25], and has successfully been used by different authors $[16,26,27,28,29,30]$. Other corrections are based on the modelling of compressibility effects of the vapour/liquid mixture in the turbulence model. Correction terms proposed by Wilcox [31] in the case of compressible flows 
have been tested for unsteady periodic cavitating flows [26]. A sensitivity analysis of constants $C_{\varepsilon 1}$ and $C_{\varepsilon 2}$, which directly influence the production and dissipation of turbulence kinetic energy, was conducted for a $k-\varepsilon$ model and a cavitating hydrofoil case [32]. Finally, filter-based methods were investigated [23] by which the sub-filter stresses are constructed directly using the filter size and the $k-\varepsilon$ turbulence closure.

The present work is part of a research aimed at developing a numerical tool devoted to cavitating flows. In previous works [33, 34], an in-house finitevolume code solving the Reynolds-Averaged Navier-Stokes (RANS) compressible equations was developed with an homogeneous approach. First computations were performed to assess numerical aspects and thermodynamic constraints on EOS. This paper is a complement work in which particular emphasis is placed on the study of turbulence models and corrections for unsteady flow, such as eddy viscosity limiters and realizability constraints. Our goals in this study are:

- to asses the sensitivity of a number of turbulence models in cavitating flows, originally developped for aerodynamic applications.

- to investigate the effect of various eddy viscosity corrections for flows involving different cavity sheet behaviours. This would help in gaining an insight into the range of predictions that we can expect with these models.

The paper is organized as follows: the theoretical formulation is summarized, including physical models and elements of the numerical methods. 
This is followed by sets of results on Venturi geometries and discussions.

\section{Governing equations and models}

The numerical simulations were carried out using an in-house CFD code solving the one-fluid compressible RANS system. First, we present the onefluid formulation for Euler equations, with the associated preconditioned method. Secondly, the complete RANS system is presented.

\subsection{The one-fluid Euler equations}

The homogeneous model assumes strong coupling of the phases and moving at the same velocity components. The phases are assumed to share the same temperature $T$ and the same pressure $P$. The evolution of the two-phase flow can be described by Euler equations that employ the representative flow properties as unknowns just as a single-phase problem. The mixture density $\rho$ is defined by:

$$
\rho=\alpha \rho_{V}+(1-\alpha) \rho_{L}
$$

where $\rho_{L}$ and $\rho_{V}$ are respectively the liquid and vapour densities. The void ratio $\alpha$ characterizes the volume of vapour in each cell: $\alpha=1$ means that the cell is completely filled by vapour; inversely, a complete liquid cell is represented by $\alpha=0$. Liquid and vapour phases are characterized by their thermodynamic properties. On each cell, the unknowns are calculated by averaging them by the volume occupied.

In order to simplify the formulation, we present below the one-dimensional 
equations, expressed in conservative variables $w=(\rho, \rho u, \rho E)$ :

$$
\begin{aligned}
\frac{\partial \rho}{\partial t}+\frac{\partial \rho u}{\partial x} & =0 \\
\frac{\partial(\rho u)}{\partial t}+\frac{\partial\left(\rho u^{2}+P\right)}{\partial x} & =0 \\
\frac{\partial(\rho E)}{\partial t}+\frac{\partial(\rho u H)}{\partial x} & =0
\end{aligned}
$$

where $E=e+u^{2} / 2$ denotes the total energy and $H=h+u^{2} / 2$ the total enthalpy; $e$ the internal energy, $h$ the enthalpy, which are related to the void ratio and the liquid and vapour values:

$$
\begin{aligned}
\rho e & =\alpha \rho_{V} e_{V}+(1-\alpha) \rho_{L} e_{L} \\
\rho h & =\alpha \rho_{V} h_{V}+(1-\alpha) \rho_{L} h_{L}
\end{aligned}
$$

To close the system, an equation of state (EOS) and a thermal relation are necessary to link the pressure and the temperature to the thermodynamic variables. Pure phases follow the stiffened gas EOS and a barotropic law is considered for the mixture.

\subsubsection{The pure-phase EOS - low Mach number preconditioning}

For pure phases, we use the convex stiffened gas equation of state (see [35]):

$$
\begin{aligned}
P(\rho, e) & =(\gamma-1) \rho(e-q)-\gamma P_{\infty} \\
P(\rho, T) & =\rho(\gamma-1) C_{v} T-P_{\infty} \\
T(\rho, h) & =\frac{h-q}{C_{p}}
\end{aligned}
$$

where $\gamma=C_{p} / C_{v}$ is the polytropic coefficient, $C_{p}$ and $C_{v}$ are thermal capacities, $q$ the energy of the fluid at a given reference state and $P_{\infty}$ is a constant 
reference pressure.

A well-known problem of compressible codes concerns the stiffness on the solution convergence when the Mach number becomes low. In this situation, the dominance of convection terms renders the system stiff and compressible solvers converge slowly. To overcome this difficulty, a preconditioned method is necessary. The physical acoustic waves are replaced by pseudo-acoustic modes that are much closer to the advective velocity, reducing the stiffness and enhancing the convergence. The preconditioned method is based on the modification of the derivative term by a premultiplication with a suitable preconditioning matrix. With the primitive variables $W=(P, u, e)$, the preconditioned Euler equations can be expressed as:

$$
P_{e}^{-1} \frac{\partial W}{\partial t}+A_{e} \frac{\partial W}{\partial x}=0
$$

We use the preconditioning matrix based on the Turkel approach [36, 37] :

$$
P_{e}=\left[\begin{array}{ccc}
\beta^{2} & 0 & 0 \\
0 & 1 & 0 \\
0 & 0 & 1
\end{array}\right] \quad ; \quad A_{e}=\left[\begin{array}{ccc}
u & \rho c^{2} & 0 \\
1 / \rho & u & 0 \\
0 & P / \rho & u
\end{array}\right]
$$

$\beta$ is a parameter on the order of the Mach number. In our study, we have chosen the form given by Choi and Merkle [38]:

$$
\beta^{2}=\min \left[\max \left(M^{2}, K M_{\infty}^{2}\right), 1\right]
$$

This form implies that there is no preconditioning used in transonic and supersonic flow regions (in the mixture). When $\beta^{2}=1$, the preconditioning 
matrix becomes the identity matrix and the system returns to its classical non preconditioned form. Moreover, for a very small flow velocity, $\beta^{2}$ is not allowed to be less than a given percentage of the freestream velocity, determined by the coefficient $K$. For inviscid computations, $K$ is near unity.

The eigenvalues of the preconditioned system are:

$$
u \quad ; \quad \lambda_{ \pm}=\frac{1}{2}\left[u\left(1+\beta^{2}\right) \pm \sqrt{\left(\beta^{2}-1\right)^{2} u^{2}+4 \beta^{2} c^{2}}\right]
$$

\subsubsection{The barotropic EOS}

The cavitation model applied in the present work is based on a sinusoidal barotropic law proposed by Delannoy et al. [6]. This law is characterized by its maximum slope $1 / c_{\text {baro }}^{2}$. The quantity $c_{\text {baro }}$ is an adjustable parameter of the model, which can be interpreted as the minimum speed of sound in the mixture.

When the pressure becomes smaller than the quantity $P_{v a p}+\Delta P$ and greater than $P_{\text {vap }}-\Delta P$, we have:

$$
P(\rho, \alpha)=P_{\text {vap }}+\left(\frac{\rho_{L}^{\text {sat }}-\rho_{V}^{\text {sat }}}{2}\right) c_{\text {baro }}^{2} \operatorname{Arcsin}(1-2 \alpha)
$$

where $\Delta P$ represents the pressure width of the law and, for a void ratio value of 0.5 , the pressure is equal to the saturation pressure $P_{v a p}$. The law introduces a small non equilibrium effect on the pressure. 
With this isothermal law, the speed of sound can be computed easily:

$$
c^{2}=\left(\frac{\partial P}{\partial \rho}\right)_{s}=\left(\frac{\partial P}{\partial \rho}\right)_{T}=\frac{c_{\text {baro }}^{2}}{2 \sqrt{\alpha(1-\alpha)}}
$$

From Eqs. (8), (13), we introduce conditions to respect the continuity of the pressure between the liquid and the mixture (when $P=P_{\text {vap }}+\Delta P$ ):

$$
\Delta P=\frac{\rho_{L}^{s a t}-\rho_{V}^{s a t}}{2} c_{\text {baro }}^{2} \frac{\pi}{2}=\rho_{L}^{\text {sat }}\left(\gamma_{L}-1\right) C_{v_{L}} T_{\text {vap }}-P_{\infty}^{L}-P_{v a p}
$$

These relations determine $c_{\text {baro }}$ for given values of saturation conditions. The continuity between the pure vapour and the mixture is not considered because, in our applications, pure gas is never reached.

The convexity of the EOS and the influence of $c_{\text {baro }}$ were studied in previous works [33]. In the present paper, the different parameters chosen for pure phases are presented in Table (1). The value of $c_{\text {baro }}$ is set to $0.472 \mathrm{~m} / \mathrm{s}$, that is correspond to a pressure width $\Delta P=175 \mathrm{~Pa}$.

\subsection{Reynolds-Averaged Navier-Stokes equations}

For turbulent computations, the compressible one-fluid RANS equations with a Favre average are used, coupled with a one- or two-equation turbulence model. For low Mach number applications, the inviscid preconditioning method presented previously is used (no viscous terms are introduced). These equations can be expressed as:

$$
P_{c}^{-1} \frac{\partial w}{\partial t}+\operatorname{div}\left(F_{c}-F_{v}\right)=S
$$




$$
w=\left(\begin{array}{c}
\rho \\
\rho V \\
\rho E \\
\rho k \\
\rho \Psi
\end{array}\right) ; F_{c}=\left(\begin{array}{c}
\rho V \\
\rho V \otimes V+p \overline{\bar{I}} \\
(\rho E+p) V \\
\rho k V \\
\rho \Psi V
\end{array}\right) \quad ; \quad F_{v}=\left(\begin{array}{c}
0 \\
\overline{\overline{\tau^{v}}}+\overline{\overline{\tau^{t}}} \\
\left(\overline{\overline{\tau^{v}}}+\overline{\overline{\tau^{t}}}\right) \cdot V-Q^{v}-Q^{t} \\
\left(\mu+\mu_{t} / \sigma_{k}\right) \operatorname{grad} k \\
\left(\mu+\mu_{t} / \sigma_{\Psi}\right) \operatorname{grad} \Psi
\end{array}\right)
$$

where $w$ denotes the conservative variables, $F_{c}$ and $F_{v}$ the convective and viscous flux densities and $S$ the source terms, which concern only the transport equations. $k$ is the turbulent kinetic energy and $\Psi$ is a turbulent variable. The exact expression of the eddy viscosity $\mu_{t}$ and the source terms depends on the turbulence model, as well as constants $\sigma_{k}$ and $\sigma_{\Psi}$.

The total stress tensor $\overline{\bar{\tau}}$ is evaluated following the Stokes hypothesis, the Newtonian law and the Boussinesq assumption. The total heat flux vector $Q$ is obtained from the Fourier law with the constant Prandtl number hypothesis.

$$
\begin{aligned}
& \overline{\bar{\tau}}=\overline{\overline{\tau^{v}}}+\overline{\overline{\tau^{t}}}=\left(\mu+\mu_{t}\right)\left[\frac{1}{2}\left(\operatorname{grad} V+(\operatorname{grad} V)^{t}\right)-\frac{2}{3}(\operatorname{div} V) \overline{\bar{I}}\right]+\frac{2}{3} k \overline{\bar{I}} \\
& Q=Q^{v}+Q^{t}=-\left(\frac{\mu}{P_{r}}+\frac{\mu_{t}}{P_{r t}}\right) C_{p} \operatorname{grad} T
\end{aligned}
$$

In the pure liquid, the viscosity is determined by an exponential law and, in pure vapour, the viscosity follows the Sutherland law. The mixture viscosity is calculated as an arithmetic mean between the liquid and the vapour viscosity:

$$
\mu_{L}(T)=\mu_{0_{L}} \exp (B / T)
$$




$$
\begin{aligned}
\mu_{V}(T) & =\mu_{0_{V}} \sqrt{\frac{T}{293}} \frac{1+T_{S} / 293}{1+T_{S} / T} \\
\mu(T, \alpha) & =\alpha \mu_{V}(T)+(1-\alpha) \mu_{L}(T)
\end{aligned}
$$

where $\mu_{0_{L}}, \mu_{0_{V}}, B$ and $T_{S}$ are constant parameters.

The mixture Prandtl number is also evaluated as an arithmetic mean between the liquid and the vapour value:

$$
P_{r}(\alpha)=\alpha P_{r_{V}}+(1-\alpha) P_{r_{L}}
$$

The turbulent Prandtl number $P_{r t}$ is set to 1 .

\subsection{Turbulence Models}

Various popular two-equation turbulence models were used in the present study: the Smith $k-l$ model (KL) $[39,40]$, the Menter SST $k-\omega$ model (KWSST) [41, 42], the high Reynolds version of the Jones-Launder $k-\varepsilon$ model (KE) [43], and the one-equation Spalart-Allmaras model (SA) [44, 45].

Turbulence models always leads to the generation of stable cavities, because very strong turbulent eddy viscosity $\mu_{t}$ inside the cavity avoids the re-entrant jet formation which plays the major role on the instability of partial sheet cavity. The link to compressibility effects on turbulence is not clear. DNS of supersonic boundary layer demonstrated the reduction in $k$ production as a consequence of compressibility $[46,47,48]$. In cavitating flows, supersonic regime is reached in the mixture area, because of the drastic diminution of the speed of sound. The detailed mechanisms of the interaction between turbulent flows and cavitation have not yet been clearly revealed especially 
for the phenomena occurring at small scales.

As a remedy to limit the turbulent viscosity, one can use a eddy- viscosity limiter in the mixture area. In the present study, we propose to test and to compare different eddy viscosity limiters: the Reboud formulation [25], the Menter SST (Shear Stress Tensor) correction [42] and Durbin realizability constraints $[49,50]$.

For the modelling of the flow close to the wall, a two-layer wall law approach is used. We assumed that wall functions are similar in a two-phase flow and in a single-phase flow. For unsteady flows, the existence of a wall law is assumed to be valid at each instant. More details concerning the wall law approach are given in [33].

\subsubsection{Reboud correction}

Reboud proposed an arbitrary limiter by introducing a function $f(\rho)$ in the computation of the turbulent viscosity for the $k-\varepsilon$ model:

$$
\mu_{t}=f(\rho) C_{\mu} \frac{k^{2}}{\varepsilon} \quad \text { with } f(\rho)=\rho_{V}+(1-\alpha)^{n}\left(\rho_{L}-\rho_{V}\right)
$$

where $n$ is a parameter fixed to 10 .

This correction is extended to other turbulence models with the same function $f(\rho)$.

\subsubsection{Menter SST correction}

The Menter correction is based on the empirical Bradshaw's assumption which binds the shear stress to the turbulent kinetic energy for two-dimensional boundary layer. The stress ratio predicted by two-equation models scales 
with the ratio of production $P_{k}$ to dissipation $\varepsilon$ as:

$$
\frac{-\overline{u^{\prime} v^{\prime}}}{k}=\sqrt{\frac{P_{k}}{\varepsilon}} \sqrt{C_{\mu}} \quad \text { with } C_{\mu}=0.09
$$

Experiments show that the quantity $-\overline{u^{\prime} v^{\prime}} / k \leq 0.3$. Menter devised his SST limiter from this inequality. The empirically based constraint is expressed as:

$$
\nu_{t}=\min \left[\frac{k}{w}, \frac{0.3 k}{\sqrt{2}|\Omega| F_{2}(y)}\right]
$$

where $F_{2}$ is a blending function that tends to zero outside the boundary layer, and $\Omega$ is the vorticity.

\subsubsection{Durbin realizability correction}

Based on the realizability principle (the variance of the fluctuating velocity components should be positive and the cross-correlations bounded by the Schwartz inequality), a minimal correction was derived for two-equation tur-

bulence models and was shown to cure the stagnation-point anomaly [49]. The condition to ensure realizability is:

$$
C_{\mu} \leq \frac{1}{s \sqrt{2}} \quad ; \quad s=\frac{k}{\varepsilon} S \quad ; \quad S^{2}=2 S_{i j} S_{i j}-\frac{2}{3} S_{k k}^{2}
$$

A weakly non-linear model was thus obtained with a $C_{\mu}$ coefficient function of the dimensionless mean strain rate :

$$
C_{\mu}=\min \left(C_{\mu}^{o}, \frac{c}{s \sqrt{2}}\right) \quad \text { with } \quad c \leq 1
$$

where $C_{\mu}^{o}$ is set to the constant value 0.09. Durbin fixed the value of the constant $c$ to 0.5 for good results in impinging jets [49]. In the present study, we chose $c=0.3$. Finally, the following relation was obtained for the $k-\varepsilon$ 
model :

$$
\mu_{t}=\rho C_{\mu} \frac{k^{2}}{\varepsilon} \quad ; \quad C_{\mu}=\min \left(C_{\mu}^{o}, \frac{c}{s \sqrt{2}}\right)
$$

It should be noted that this correction is similar to the SST formula by replacing $\Omega$ with $S$. Yet, the Durbin correction is established with mathematical concepts and is available for three-dimensional flows whereas the SST correction is based on an empirical two-dimensional hypothesis.

\section{Numerical methods}

The numerical simulations were carried out using an implicit CFD code solving the RANS/turbulent systems for multi-domain structured meshes. This solver is based on a cell-centered finite-volume discretization.

\subsection{Spatial discretization}

For the mean flow, the convective flux density vector on a cell face is computed with the Jameson scheme [51] in which the dispersive error is cancelled. The artificial viscosity includes a second-order dissipation term $D_{2}$ and a fourth-order dissipation term $D_{4}$, which involve two tunable parameters $k^{(2)}$ and $k^{(4)}$.

The viscous terms are discretized by a second-order space-centered scheme. For the turbulence transport equations, the upwind Roe scheme [52] is used to obtain a more robust method. The second-order accuracy is obtained by introducing a flux-limited dissipation [53]. 


\subsection{Temporal discretization}

Time integration is achieved using a low-cost implicit method [54]. The implicit method consists in solving, at each time step, a system of equations arising from the linearization of a fully implicit scheme. The main advantage of this method is that the storage of the Jacobian matrix is completely eliminated, which leads to a low-storage algorithm. More details are given in [33].

For the turbulence transport equations, the diffusive flux Jacobian matrix is replaced by its spectral radius. The source term needs special treatment [55]. Only the negative part of the source term Jacobian matrix is considered and replaced by its spectral radius. The system obtained is solved with a linealternated Jacobi relaxation algorithm.

\subsection{Inlet and outlet boundary conditions}

The numerical treatment of boundary conditions is a key point in the case of propagations of large amplitude pressure waves in the computation domain. It is based on the use of the preconditioned characteristic relations. The number of variables to impose at boundaries is given by the number of positive characteristics. The characteristic relations obtained for the preconditioned system, in two-dimensional flows, are:

$$
\begin{aligned}
-c^{2}\left(\rho^{c}-\rho^{s}\right)+\left(P^{c}-P^{s}\right) & =0 \\
V_{t}^{c}-V_{t}^{s} & =0 \\
\left(\lambda_{+}-V_{n}\right)\left(P^{c}-P^{s}\right)+\rho \beta^{2} c^{2}\left(V_{n}^{c}-V_{n}^{s}\right) & =0 \\
\left(\lambda_{-}-V_{n}\right)\left(P^{c}-P^{s}\right)+\rho \beta^{2} c^{2}\left(V_{n}^{c}-V_{n}^{s}\right) & =0
\end{aligned}
$$


The variables with superscript $c$ are those to be computed at the boundary. Variables with superscript $s$ are obtained by the current numerical scheme. $V_{t}$ and $V_{n}$ are the tangential and normal components of the mean velocity, respectively.

At inflow, four variables have to be imposed: we chose the stagnation pres-

sure $P_{i}$, the stagnation temperature $T_{i}$, and the direction of the velocity. A Newton algorithm can be used to compute the pressure with the characteristic relation (32) and the conservative variables can be evaluated at the boundary.

At outflow, only one variable is imposed: the static pressure was chosen. The conservative variables are computed with three characteristic relations (29)-(31).

We assumed that inlet and outlet areas are in a pure liquid region. No cavitation appears in these boundaries. For cavitating computations, we iterate on the outlet pressure to get a given inlet pressure value.

\section{Computational results on Venturi geometries}

The Venturi test section of the CREMHyG (Centre d'Essais de Machines Hydrauliques de Grenoble) cavitation tunnel was sized and designed to simulate cavitating flows developing on the blades of space turbopump inducers. Two geometries have been tested: a first one with quasi-stable cavities and a second with periodic oscillating cavities. 


\subsection{Case 1: quasi-stable cavity}

\subsubsection{Experimental conditions [56]}

The first Venturi is characterized by a divergence angle of $4^{\circ}$, illustrated in Fig. 1. The edge forming the throat of the Venturi is used to fix the separation point of the cavitation cavity. The geometrical data are:

Inlet section: $S_{i}=50 \times 44 \mathrm{~mm}^{2}$ (where the reference pressure is measured);

Throat section: $S_{\text {throat }}=43.7 \times 44 \mathrm{~mm}^{2}$;

Length of the test section (chord): $L_{r e f}=252 \mathrm{~mm}$.

This geometry is equipped with five probing holes to take various measurements such as the local void ratio and instantaneous local speed (Fig. 1). The mean wall pressures were also measured for nine probed stations including the five stations inside the cavitation sheet (where void ratio and velocity measurements were performed) and also four other stations placed downstream in the wake of the sheet.

The selected operating point is characterized by the following physical parameters [56]:

$U_{\text {inlet }}=10.8 \mathrm{~m} / \mathrm{s}:$ the inlet velocity

$Q=0.02375 \mathrm{~m}^{3} / \mathrm{s}$ : the flow imposed in the circuit

$P_{\text {inlet }}=35000 \mathrm{~Pa}:$ the pressure in the inlet section

$\sigma_{\text {inlet }}=\frac{P_{\text {inlet }}-P_{\text {vap }}}{0.5 \rho U_{\text {inlet }}^{2}} \simeq 0.55:$ the cavitation parameter in the inlet section

$T_{\text {ref }} \simeq 293 \mathrm{~K}:$ the reference temperature

$R e_{L_{r e f}}=\frac{U_{\text {inlet }} L_{r e f}}{\nu}=2.710^{6}:$ the Reynolds number

With these parameters, a cavity length $L$ ranging from $70 \mathrm{~mm}$ to $85 \mathrm{~mm}$ has 
been obtained. The experimental views for this geometry show a relatively stable cavity behaviour, as shown in Fig. 2. A stable or quasi-stable cavity is characterized by an almost constant length, although the closure region always fluctuates with the existence of a re-entrant jet and little vapour cloud shedding. For this geometry, no periodic cycles with large shedding were observed.

\subsubsection{Mesh}

The grid is a H-type topology. It contains 251 nodes in the flow direction and 62 in the orthogonal direction. A special contraction of the mesh is applied in the main flow direction just after the throat to better simulate the two-phase flow area (Fig. 3). The $y^{+}$values of the mesh, at the center of the first cell, vary between 12 and 27 for a non cavitating computation.

According to the study of mesh dependence presented in [57], this grid size is adequate to simulate cavitation sheets in such Venturi type section.

\subsubsection{Numerical parameters}

For the non cavitating regime, computations are started from an uniform flow-field using a local time step. The numerical parameters used are:

- the CFL number, 10

- the preconditioned parameter, $K=1$

- Jacobi iterations for the implicit stage, 15

- the two coefficients of the artificial dissipation, $k^{(2)}=0$ and $k^{(4)}=0.032$

- the farfield value of turbulent viscosity, $\mu_{t_{\infty}}=0.1 \mu_{\infty}$

- the farfield value of turbulent kinetic energy, $k_{\infty}=0.0045 \mathrm{~m}^{2} / \mathrm{s}^{2}$

- the farfield value of specific dissipation, $\omega_{\infty}=45000 \mathrm{~s}^{-1}$ 
For the unsteady cavitating regime, computations are performed with the dual time stepping method and are started from the non cavitating numerical solution. The numerical parameters are:

- the dimensionless time step, $\Delta t^{*}=\frac{\Delta t U_{\text {inlet }}}{L_{\text {ref }}}=9.7510^{-3}$

- sub-iterations of the dual time stepping method, 100

- the CFL number, 0.5

- the preconditioned parameter, $K=3$

- Jacobi iterations for the implicit stage, 15

- the two coefficients of the artificial dissipation: $k^{(2)}=1$. and $k^{(4)}=0.04$.

\subsubsection{Global analyses}

Different calculations were performed by considering different turbulence models, summarized in Table (2). The goal was to obtain a quasi-stable cavitation sheet whose length varied between $70-85 \mathrm{~mm}$ with a re-entrant jet. The time of simulation is between 4 and $5 \mathrm{~s}$.

Firstly, all computations were able to capture such cavity sheet. The tested limiters: Reboud, SST and realizability constraints make possible the simulation of unsteady behaviours.

Secondly, the SST Menter model captured a periodic self-oscillating cavity whereas all other models simulated an aperiodic cavity. Moreover, the $\sigma_{\text {inlet }}$ value is around 0.62 , a larger value in comparison with the experimental value equal to 0.55 .

The dimensionless vapour volume evolutions (the volume is divided by $L_{r e f}^{3}$ ) are plotted in Fig. 4 for the Smith $k-l$ model, and in Fig. 5 for the Menter model. We can observe the periodic signal obtained with the Menter model, 
the frequency is around $10 \mathrm{~Hz}$. For all other models, a direct Fourier transformation (DFT) of the vapour volume did not reveal any frequency.

\subsubsection{Velocity and void ratio profiles}

Local analyses concern void ratio and velocity profile comparisons inside the cavity. The experimental void ratio and velocity profiles are obtained for five stations by a double optical probe (Fig. 1). The velocity is evaluated as the most probable value and the void ratio is obtained from the signal of the double optical probe using a post-processing algorithm. The relative uncertainty on the void ratio measurement was estimated at roughly $15 \%$ [56]. Numerical values were obtained by a time-averaged treatment.

Figure 6 shows the longitudinal velocity profiles for the experiments and the five computations. The overall agreement seems good between the experimental data and the simulations. For stations 1 and 2, no re-entrant jet phenomena occurs in the experiment. Yet, the Menter and the KE Reboud models simulated a re-entrant jet. One possible explanation is that the $\mu_{t}$ limiter is too strong, which allows the development of the jet. Associated with the Durbin realizability constraints, the $k-\varepsilon$ shows a better behaviour. Further downstream, for stations 3, 4 and 5, experimental observation indicates a recirculating behaviour with a re-entrant jet extending roughly through half the sheet thickness (see Figure 7 to get the sheet thickness). According to experiments, this flow configuration is smoothly time fluctuating. This recirculating behaviour with a re-entrant jet is well simulated by all computations. At station 3, the thickness of the recirculating area is correctly estimated by all calculations, except by the KE Reboud model. At 
station 5, large discrepancies appear between models.

Figure 7 illustrates experimental and numerical results concerning the void ratio profiles. For the first station, close to the throat, the vaporization phenomenon is clearly represented. This is a relatively strong effect, and the void ratio value is almost equal to 0.9 near the wall. For all computations, the cavity thickness is very well estimated.

Downstream, at the second station, the void ratio is higher (around 96\%). The distribution is similar to that obtained for station 1, with a correct estimation of the sheet thickness. The maximum value of the void ratio is under-estimated with the Menter model. The void ratio profile computed with the KE Reboud model presents a non monotonic profile.

From the third station, the re-entrant jet becomes noticeable, as observed before in the velocity field analyses. The void ratio value at the wall is largely over-estimated by all computations. As noted above, the cavity thickness is well predicted by all calculations.

At station 4, large discrepancies are observed for the cavity thickness, the maximum and the wall void ratio values. Only the KE Reboud model gives a wall value close to the experimental one.

At the last station, all computations over-predicted the maximum void ratio value.

\subsubsection{Wall pressure and RMS fluctuations}

The wall pressure distribution is plotted in Fig. 8 versus the distance $x-x_{\text {inlet }}$. The first five data are located inside the cavity (where the void ratio and velocity profiles are measured). For all computations, the pressure remains at 
an almost constant value $P_{v a p}$ in the cavity. Downstream, large discrepancies are notable between models. The re-compression is under-estimated with the $k-\varepsilon$ models, especially the realizable version.

The Root Mean Square (RMS) wall pressure fluctuations are plotted in Fig. 9 and 10 versus the distance $x-x_{\text {inlet }}$. The pressure fluctuation is divided by the time-averaged pressure Pav. For all computations, the statistical treatment was performed on a simulation time of $1 \mathrm{~s}$. For the experiment, the time is greater than 3 minutes. Experimental data indicate an augmentation of pressure fluctuation at the end of the cavity sheet, with a peak located at the fifth station.

All models over-estimated the fluctuation intensity at the end of the cavity. The peak position varies between models. With the Spalart-Allmaras and the Menter models, a very intense peak is present downstream the fifth station, whereas the peak obtained with the $k-l$ and $k-\varepsilon$ Reboud models is upstream. The realizable $k-\varepsilon$ model provides fluctuations in better agreement with experimental data. Yet, for the last stations, fluctuations are over-predicted. That is also the case for the Smith model with the Reboud correction.

\subsubsection{Turbulent eddy viscosity profiles}

Figure 11 compares time-averaged profiles of the ratio $\mu_{t} / \mu$, at the five stations, obtained with a standard Spalart-Allmaras model and the same model coupled with the Reboud correction. With the standard model, the computed cavity is steady. We can clearly observe the drastic decrease of $\mu_{t}$ due to the Reboud correction. The value of $\mu_{t} / \mu$ is close to zero in a part of the cavity, except at station 5 . The maximum value is reduced by a factor close 
to 10 .

Figure 12 compares $\mu_{t} / \mu$ profiles obtained with a standard $k-\varepsilon$ model and both corrected KE models. With the standard model, the cavity computed is weakly unsteady, with a very small fluctuating area at the end of the cavity. As commented previously, the Reboud correction reduces drastically the turbulent eddy viscosity, except at station 5 . The reduction provided by the realizability constraints is less important. At station 5, both corrections give a similar reduction. The Reboud limiter is certainly too strong, which allows the re-entrant jet to flow upstream (Fig 6). It seems that the reduction generated by realizability constraints is enough to capture unsteady quasi stable sheet.

\subsubsection{Density gradient and Q-criterion}

A qualitative description of the dynamic of cavity sheets is proposed with the plotting of the contours of the density gradient modulus (Schlieren-like visualizations) and the iso-lines of the Q-criterion.

Positive values of the Q-criterion, defined as the second invariant of the velocity gradient tensor $\frac{\partial u_{i}}{\partial x_{j}}([58])$,

$$
Q=\frac{1}{2}\left[\left(\frac{\partial u_{i}}{\partial x_{i}}\right)^{2}-\frac{\partial u_{i}}{\partial x_{j}} \frac{\partial u_{j}}{\partial x_{i}}\right]
$$

are used to identify vortices and local rotational areas. A dimensionless quantity is built with the inlet velocity and the reference length. Iso-lines levels vary between 0.01 and 1 . 
Figure 13 illustrates both contours of density gradients (on the left) and the dimensionless Q-criterion (on the right), at five instants, obtained with the SA Reboud model. The cavity interface is well exhibited by the density gradient contours. Around the abscissa $x=0.3 \mathrm{~m}$, we can observe the attached cavity closure and downstream the fluctuating recirculation with two-phase structures shedding. The generated shear layer with vortical clouds of cavitation is clearly shown with the Q-criterion. For this case, the wall jet is probably not intense enough to break the sheet and to induce cyclic clouds shedding.

Figure 14 shows both contours of density gradients (on the left) and the dimensionless Q-criterion (on the right), during one period, for the Menter SST model. The dynamic of the cavitation sheet is quite different in comparison with the SA Reboud model. On the top, we can distinguished the interface of the attached cavity sheet (around the abscissa $x=0.2 \mathrm{~m}$ ), and downstream two large cavities with interfaces located around $x=0.45 \mathrm{~m}$. These two cavities collapse, the length decreases while the re-entrant jet develops and produces a new cavity. The cycle is initiated. On the right, with the Q-criterion, we can observe the two shear layers associated with the two cavities, and vortices shed in the flow downstream the sheet.

\subsection{Case 2: periodic self-oscillating cavity}

\subsubsection{Experimental conditions [16]}

The Venturi is characterized by a divergence angle of $8^{\circ}$, illustrated in Fig. 15. The geometrical data are:

Inlet section: $S_{i}=50 \times 44 \mathrm{~mm}^{2}$ (where the reference pressure is measured); 
Throat section: $S_{\text {throat }}=33.5 \times 44 \mathrm{~mm}^{2}$;

Length of the test section (chord): $L_{r e f}=224 \mathrm{~mm}$.

This geometry is equipped with three probing holes to take various measurements such as the local void ratio and instantaneous local velocity (Fig. 16).

The selected operating point is characterized by the following physical parameters [16]:

$U_{\text {inlet }}=7.04 \mathrm{~m} / \mathrm{s}:$ the inlet velocity

$Q=0.0155 \mathrm{~m}^{3} / \mathrm{s}$ : the flow imposed in the circuit

$P_{\text {inlet }}=55000 \mathrm{~Pa}:$ the pressure in the inlet section

$\sigma_{\text {inlet }}=\frac{P_{\text {inlet }}-P_{\text {vap }}}{0.5 \rho U_{\text {inlet }}^{2}} \simeq 2.15 \pm 0.06$ : the cavitation parameter in the inlet section

$T_{\text {ref }} \simeq 293 \mathrm{~K}:$ the reference temperature

$R e_{L_{\text {ref }}}=\frac{U_{\text {inlet }} L_{r e f}}{\nu}=1.5710^{6}$ : the Reynolds number

With these parameters, an unsteady cavity with quasi-periodic fluctuations of the attached sheet and vapour clouds shedding has been obtained. Fig. 17 shows a instantaneous photograph of the cavity with a large structure shedding. The maximum length $L$ of the attached cavity is about $45 \mathrm{~mm}$. The vapour shedding frequency is close to $45 \mathrm{~Hz}$ estimated from Fourier transformations of unsteady pressure signal.

\subsubsection{Mesh}

The grid is a H-type topology. It contains 250 nodes in the flow direction and 61 in the orthogonal direction. A special contraction of the mesh is applied in the main flow direction just after the throat to better simulate the two-phase 
flow area (Fig. 18). The $y^{+}$values of the mesh, at the center of the first cell, vary between 9 and 31 for a non cavitating computation.

\subsubsection{Numerical parameters}

This test case is difficult to compute because inlet conditions fluctuate in time and large amplitude pressure waves propagate in the domain. Due to the stiffness of the case, the preconditioning parameter is increased: $K=5$, and the dimensionless time step is reduced: $\Delta t^{*}=\frac{\Delta t U_{\text {inlet }}}{L_{\text {ref }}}=6.410^{-4}$.

\subsubsection{Global analyses}

Different calculations were performed by considering different turbulence models, summarized in Table (3). The goal was to obtain a periodic cavitation sheet with a frequency close to $45 \mathrm{~Hz}$. The simulation time is around $3 \mathrm{~s}$, except for the Menter model for which the time is only 2 seconds.

Firstly, it was impossible to obtain converge results with the $k-\varepsilon$ realizable model. This model generated large vapour shedding with large pressure variations in the computation domain, leading to the divergence of the simulation. On the other hand, all other models were able to capture an unsteady cavity sheet with two-phase structures shedding.

Secondly, all models did not capture a periodic self-oscillating cavity. The dimensionless vapour volume evolutions are plotted in Fig. 19 for the SpalartAllmaras Reboud model, in Fig. 20 for the Smith Reboud model, and in Fig. 21 for the Menter SST model. For all models, a direct Fourier transformation (DFT) of the vapour volume signal was performed to evaluate the frequency. Fig. 22, 23 and 24 present the frequency results. Both Spalart- 
Allmaras and Jones-Launder models captured a quasi cyclic phenomenon, with a frequency close to $45 \mathrm{~Hz}$ (a smaller one with the $\mathrm{KE}$ model and a higher with the SA model). With the Smith model, no frequency appeared. The Menter SST model simulated a phenomenon with two frequencies: 36 and $50 \mathrm{~Hz}$. A possible explanation is that the attached cavity sheet oscillates at a different frequency of the clouds shedding.

\subsubsection{Velocity and void ratio profiles}

The experimental data are obtained by a double optical probe [16]. Identically to the first case, the velocity is evaluated as the most probable value and the void ratio is obtained with a post-processing algorithm. For the velocity profiles, experimental data are given for the first two stations. All numerical values were obtained by a time-averaged treatment.

Figure 25 shows the evolution of the longitudinal velocity for the experiments and all computations. All models provided similar results. The re-entrant jet phenomenon is well captured by all models.

Figure 26 illustrates experimental and numerical results concerning the void ratio profiles. Experimental values are weak, even at station 1. For this station, all models largely over-predicted the void ratio, up to a factor of 4. The best result is obtained with the Menter SST model. At station 2, except for the Spalart Reboud model, the void ratio is still over-predicted. For the last station, all models associated with the Reboud correction underestimated the cavity length and provided a pure liquid profile. On the other hand, the Menter SST model captured mixture clouds, but the void ratio 
values are too important.

\subsubsection{Density gradient and Q-criterion}

Figure 27 illustrates both contours of density gradients modulus (on the left) and the dimensionless Q-criterion (on the right), during one period, obtained with the SA Reboud model. Q-criterion iso-lines levels vary between 0.025 and 0.5. We can observe a small attached cavity, and downstream the fluctuating recirculation with two-phase structures shedding. These shedding are not extended and are rapidly eliminated. In comparison with the experimental visualizations, the attached cavity and the clouds shedding are under-estimated. Similar results were obtained with the Smith and the JonesLaunder models associated with the Reboud correction.

Figure 28 plots both contours of density gradients (on the left) and the dimensionless Q-criterion (on the right), during one period, obtained with the Menter SST model. Results are quite different to those obtained with the Spalart-Allmaras model. There remains also a small attached cavity near the throat. Clouds shedding are more extended. The solution given by the SST model seems in better qualitative agreement with the experimental data.

\section{Conclusion}

A two-phase one-fluid compressible CFD solver was applied to simulate unsteady Venturi cavitating flows. Turbulent eddy viscosity limiters for transport-equation models were outlined, including an arbitrary limiter, the Menter SST correction and realizability constraints of Durbin. The presented 
numerical results demonstrated the great influence of such corrections to capture unsteady behaviours of cavity sheets on Venturi geometries. Global and local analyses of flows were proposed based on void ratio and velocity profiles.

First computations were performed to study an aperiodic quasi-stable cavitation sheet. Compared to previous works done in the same geometry, more accurate and reliable results were obtained. The unsteady behaviour of the cavity is well described, in close agreement with the experimental data. Simulations demonstrated the determinant role of the use of a viscosity limiter. The Reboud correction associated with the Spalart-Allmaras and the JonesLaunder models provided a quasi stable sheet with a significant re-entrant jet. Similar results were obtained with the realizable $k-\varepsilon$ models. The Menter SST model captured a different solution with a low-frequency periodic cavitation sheet.

Secondly, computations were performed to study a periodic unstable cavitation sheet, with the break-off of the sheet and two-phase structures shedding. The Reboud correction associated with all tested turbulence models provided an unstable cavity with small shedding. The computed vapour volume showed irregular behaviours, more or less periodic. With the Smith $k-l$ model, no frequency appeared. With the Menter SST model, two frequencies are revealed. In terms of local profiles and the qualitative dynamic of the cavity, the Menter SST model captured the solution closest to the experimental data.

Additional works are in progress to investigate other turbulence models associated with other corrections, hydrofoil geometries, and to pursue compar- 
ative analyses between numerical and experimental studies. 
[1] S. Clerc, Numerical simulation of the homogeneous equilibrium model for two-phase flows, Journal of Computational Physics 161 (1) (2000) $354-375$.

[2] F. D. Vuyst, J. Ghidaglia, G. L. Coq, On the numerical simulation of multiphase water flows with changes of phase and strong gradients using the Homogeneous Equilibrium Model, Int. Journal on Finite Volumes 2 (1) (2005) 1-36.

[3] Y. Ventikos, G. Tzabiras, A numerical method for the simulation of steady and unsteady cavitating flows, Computers \& Fluids 29 (1) (2000) 63-88.

[4] T. Barberon, P. Helluy, Finite volume simulation of cavitating flows, Computers \& Fluids 34 (7) (2005) 832-858.

[5] O. Coutier-Delgosha, J. Reboud, Y. Delannoy, Numerical simulation of the unsteady behaviour of cavitating flow, Int. Journal for Numerical Methods in Fluids 42 (2003) 527-548.

[6] Y. Delannoy, J. Kueny, Two phase flow approach in unsteady cavitation modelling, in: Cavitation and Multiphase Flow Forum, ASME-FED, vol. 98, pp153-158, 1990.

[7] B. Pouffary, R. F. Patella, J. Reboud, P. Lambert, Numerical simulation of 3D cavitating flows: analysis of cavitation head drop in turbomachinery, Journal of Fluids Engineering 130 (6) (2008) 061301.

[8] T. Liu, B. Khoo, W. X. WF, Isentropic one-fluid modelling of unsteady 
cavitating flow, Journal of Computational Physics 201 (1) (2004) 80108.

[9] J. Moreau, O. Simonin, C. Habchi, A numerical study of cavitation influence on diesel jet atomisation, in: 19th annual meeting of the institute for liquid atomization and spray systems, Notthingham, England, 2004.

[10] S. Schmidt, I. Sezal, G. Schnerr, Compressible simulation of high-speed hydrodynamics with phase change, in: European Conference on Computational Fluid Dynamics ECCOMAS 2006, Delft, The Netherlands, 2006.

[11] C. Song, Current status of cfd for cavitating flows, in: 9th International Symposium on Transport Phenomena and Dynamics of Rotating Machinery, Honolulu, Hawai, 2002.

[12] E. Sinibaldi, F. Beux, M. Salvetti, A numerical method for 3D barotropic flows in turbomachinery, Flow Turbulence Combustion 76 (2006) 371381.

[13] J. Edwards, R. Franklin, Low-diffusion flux splitting methods for real fluid flows with phase transition, AIAA Journal 38 (9) (2000) 1624-1633.

[14] R. Saurel, R. Abgrall, A simple method for compressible multifluid flows, SIAM Journal of Scientific Computing 21 (3) (1999) 1115-1145.

[15] V. Ahuja, A. Hosangadi, S. Arunajatesan, Simulations of cavitating flows using hybrid unstructured meshes, Journal of Fluids Engineering 123 (2) (2001) 331-340. 
[16] R. F. Patella, S. Barre, J. Reboud, Experiments and modelling of cavitating flows in Venturi : part II, unsteady cavitation, in: 6th International Symposium on Cavitation CAV2006, Wageningen, The Netherlands, 2006.

[17] U. Iben, F. Wrona, C. Munz, M. Beck, Cavitation in hydraulic tools based on thermodynamic properties of liquid and gas, Journal of Fluids Engineering 124 (4) (2002) 1011-1017.

[18] R. Kunz, D. Boger, D. Stinebring, T. Chyczewski, J. Lindau, H. Gibeling, S. Venkateswaran, T. Govindan, A preconditioned navier-stokes method for two-phase flows with application to cavitation prediction, Computers \& Fluids 29 (8) (2000) 849-875.

[19] J. Lindau, R. Kunz, D. Boger, D. Stinebring, H. Gibeling, High Reynolds number, unsteady, multiphase CFD modeling of cavitating flows, Journal of Fluids Engineering 124 (3) (2002) 124-607.

[20] I. Senocak, W. Shyy, A pressure-based method for turbulent cavitating flow computations, Journal of Computational Physics 176 (2) (2002) $363-383$.

[21] B. Shin, Y. Iwata, T. Ikohagi, Numerical simulation of unsteady cavitating flows using a homogeneous equilibrium model, Computational Mechanics 30 (2003) 388-395.

[22] A. Singhal, M. Athavale, H. Li, Y. Jiang, Mathematical basis and validation of the full cavitation model, Journal of Fluids Engineering 124 (3) (2002) 617-624. 
[23] J. Wu, G. Wang, W. Shyy, Time-dependent turbulent cavitating flow computations with interfacial transport and filter-based models, Int. Journal for Numerical Methods in Fluids 49 (7) (2005) 739-761.

[24] Y. Utturkar, J. Wu, G. Wang, W. Shyy, Recent progress in modelling of cryogenic cavitation for liquid rocket propulsion, Progress in Aerospace Sciences 41 (2005) 558-608.

[25] J.-L. Reboud, B. Stutz, O. Coutier, Two-phase flow structure of cavitation: experiment and modelling of unsteady effects, in: $3^{\text {rd }}$ International Symposium on Cavitation CAV1998, Grenoble, France, 1998.

[26] O. Coutier-Delgosha, R. Fortes-Patella, J.-L. Reboud, Simulation of unsteady cavitation with a two-equation turbulence model including compressibility effects, Journal of Turbulence 3 (58).

[27] Y. Chen, C. Lu, L. Wu, Modelling and computation of unsteady turbulent cavitation flows, Journal of Hydrodynamics 18 (5) (2006) 559-566.

[28] L. Zhou, Z. Wang, Numerical simulation of cavitation around a hydrofoil and evaluation of a rng $k-\varepsilon$ model, Journal of Fluid Engineering 130 (1) (2008) 011302.

[29] V. Srinivasan, A. Salazar, K. Saito, numerical simulation of cavitation dynamics using a cavitation-induced-momentum-defect (cimd) correction approach, Applied Mathematical Modelling 33 (2009) 1529-1559.

[30] J. Seo, S. Lele, Numerical investigation of cloud cavitation and cavitation noise on a hydrofoil section, in: $7^{\text {th }}$ International Symposium on Cavitation CAV2009, Ann Arbor, USA, 2009. 
[31] D. Wilcox, Turbulence modeling for CFD, DCW Industries Inc. (1998).

[32] R. Vaidyanathan, I. Senocak, J. Wu, W. Shyy, Sensitivity evaluation of a transport-based turbulent cavitation model, Journal of Fluids Engineering 125 (5) (2003) 447-458.

[33] E. Goncalves, R. F. Patella, Numerical simulation of cavitating flows with homogeneous models, Computers \& Fluids 38 (9) (2009) 16821696.

[34] E. Goncalves, R. F. Patella, Numerical study of cavitating flows with thermodynamic effect, Computers \& Fluids 39 (1) (2010) 99-113.

[35] O. L. Metayer, J. Massoni, R. Saurel, Elaborating equations of state of a liquid and its vapor for two-phase flow models, Int. Journal of Thermal Sciences 43 (2004) 265-276.

[36] H. Guillard, C. Viozat, On the behaviour of upwind schemes in the low Mach number limit, Computers \& Fluids 28 (1) (1999) 63-86.

[37] E. Turkel, Preconditioned methods for solving the incompressible and low speed compressible equations, Journal of Computational Physics 172 (2) (1987) 277-298.

[38] Y. Choi, C. Merkle, The application of preconditioning to viscous flows, Journal of Computational Physics 105 (2) (1993) 207-223.

[39] B. Smith, The $k-k l$ turbulence model and wall layer model for compressible flows, in: AIAA 90-1483, $21^{\text {st }}$ Fluid and Plasma Dynamics Conference - Seattle, Washington, 1990. 
[40] B. Smith, A near wall model for the $k-l$ two equation turbulence model, in: AIAA 94-2386, 25 $5^{\text {sh }}$ Fluid Dynamics Conference - Colorado Springs, Colorado, 1994.

[41] F. Menter, Zonal two equation $k-\omega$ turbulence models for aerodynamic flows, in: AIAA 93-2906, 24th Fluid Dynamics Conference - Orlando, Florida, 1993.

[42] F. Menter, Two-equation eddy-viscosity turbulence models for engineering applications, AIAA Journal 32 (8) (1994) 1598-1605.

[43] W. Jones, B. Launder, The prediction of laminarization with a twoequation model of turbulence, Int. J. Heat Mass Transfer 15 (1972) $301-314$.

[44] P. Spalart, S. Allmaras, A one-equation turbulence model for aerodynamic flows, in: AIAA 92-0439, 30 ${ }^{\text {th }}$ Aerospace Sciences Meeting Reno, Nevada, 1992.

[45] P. Spalart, S. Allmaras, A one-equation turbulence model for aerodynamic flows, La Recherche Arospatiale (1) (1994) 5-21.

[46] P. Huang, G. Coleman, P. Bradshaw, Compressible turbulent channel flows: Dns results and modelling, Journal of Fluid Mechanics 305 (1995) $185-218$.

[47] S. Sarkar, The stabilizing effect of compressibility in turbulent shear flow, Journal of Fluid Mechanics 282 (1995) 163-186. 
[48] R. Lechner, J. Sesterhenn, R. Friedrich, Turbulent supersonic channel flow, Journal of Turbulence 2 (1).

[49] P. Durbin, On the $k-\varepsilon$ stagnation point anomaly, International Journal of Heat and Fluid Flow 17 (1) (1996) 89-90.

[50] P. Durbin, Limiters and wall treatments in applied turbulence modeling, Fluid Dyn. Research 41 (1) (2009) 012203.

[51] A. Jameson, W. Schmidt, E. Turkel, Numerical solution of the Euler equations by finite volume methods using Runge-Kutta time stepping schemes, in: AIAA Paper 81-1259, 1981.

[52] P. Roe, Approximate Riemann solvers, parameters vectors, and difference schemes, Journal of Computational Physics 43 (1981) 357-372.

[53] S. Tatsumi, L. Martinelli, A. Jameson, Flux-limited schemes for the compressible Navier-Stokes equations, AIAA Journal 33 (2) (1995) 252261.

[54] H. Luo, J. Baum, R. Lohner, A fast, matrix-free implicit method for compressible flows on unstructured grids, Journal of Computational Physics 146 (2) (1998) 664-690.

[55] B. Merci, J. Steelant, J. Vierendeels, K. Riemslagh, E. Dick, Computational treatment of source terms in two-equation turbulence models, AIAA Journal 38 (11) (2000) 2085-2093.

[56] S. Barre, J. Rolland, G. Boitel, E. Goncalves, R. F. Patella, Experiments 
and modelling of cavitating flows in Venturi: attached sheet cavitation, European Journal of Mechanics B/Fluids 28 (2009) 444-464.

[57] O. Coutier-Delgosha, R. F. Patella, J. Reboud, N. Hakimi, C. Hirsch, Numerical simulation of cavitating flow in 2D and 3D inducer geometries, Int. Journal for Numerical Methods in Fluids 48 (2005) 135-167.

[58] J. Hunt, C. Wray, P. Moin, Eddies, streams, and convergence zones in turbulent flows, Tech. rep., Center for Turbulence Research, CTR-S88 (1988). 
Table 1: Parameters of the pure phases EOS.

\begin{tabular}{llllllll}
\hline & $\gamma$ & $P_{\infty}(\mathrm{Pa})$ & $\mathrm{q}(\mathrm{J} / \mathrm{kg})$ & $C_{p}(\mathrm{~J} / \mathrm{K} . \mathrm{kg})$ & $c(\mathrm{~m} / \mathrm{s})$ & $\rho_{\text {sat }}\left(\mathrm{kg} / \mathrm{m}^{3}\right)$ & $e_{\text {sat }}(\mathrm{J} / \mathrm{kg})$ \\
\hline liquid & 1.01 & $1.21110^{7}$ & $-0.114210^{7}$ & 4183 & 110.7 & 998.16 & 83779 \\
vapor & 1.32 & 0 & $0.198510^{7}$ & 1883 & 423 & 0.0173 & $2.40210^{6}$ \\
\hline
\end{tabular}


Table 2: Unsteady computations, $4^{\circ}$ Venturi.

\begin{tabular}{llll}
\hline case & turb model & $\sigma_{\text {inlet }}$ & comments \\
\hline 1 & SA + Reboud & 0.57 & aperiodic quasi stable sheet \\
2 & KL + Reboud & 0.56 & aperiodic quasi stable sheet \\
3 & KE + Reboud & 0.56 & aperiodic quasi stable sheet \\
4 & KE + Durbin & 0.55 & aperiodic quasi stable sheet \\
5 & KWSST & 0.62 & periodic quasi stable sheet \\
\hline
\end{tabular}


Table 3: Unsteady computations, $8^{\circ}$ Venturi.

\begin{tabular}{llll}
\hline case & turb. model & time-averaged $\sigma_{\text {inlet }}$ & comments \\
\hline 1 & SA + Reboud & 2.07 & periodic sheet, $49 \mathrm{~Hz}$ \\
2 & KL + Reboud & 2.04 & aperiodic sheet \\
3 & KE + Reboud & 2.07 & periodic sheet, $40 \mathrm{~Hz}$ \\
4 & KE + Durbin & 2.04 & divergence \\
5 & KWSST & 2.06 & bi-periodic sheet, 36 and $50 \mathrm{~Hz}$ \\
\hline
\end{tabular}




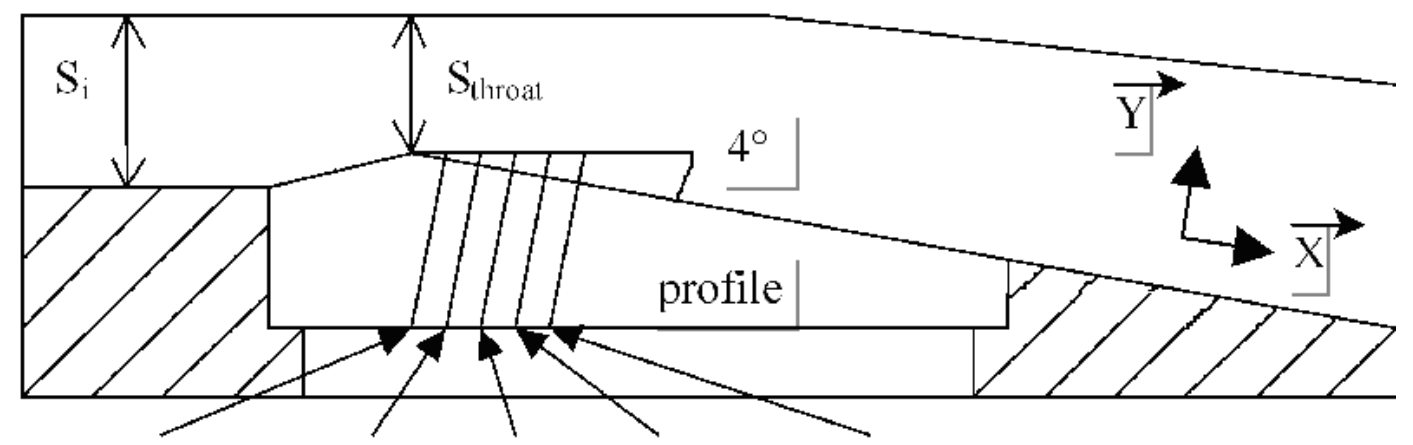

Station 1 Station 2 Station 3 Station 4 Station 5

Figure 1: Schematic view of the $4^{\circ}$ Venturi profile. 


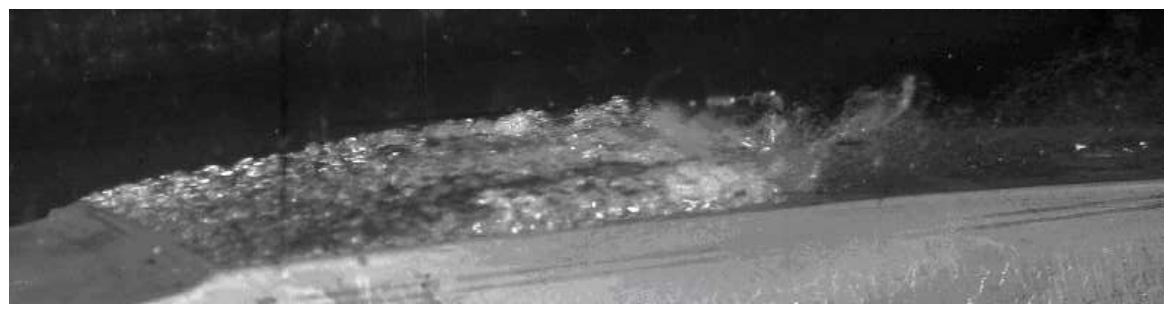

Figure 2: Photograph of the cavity. 


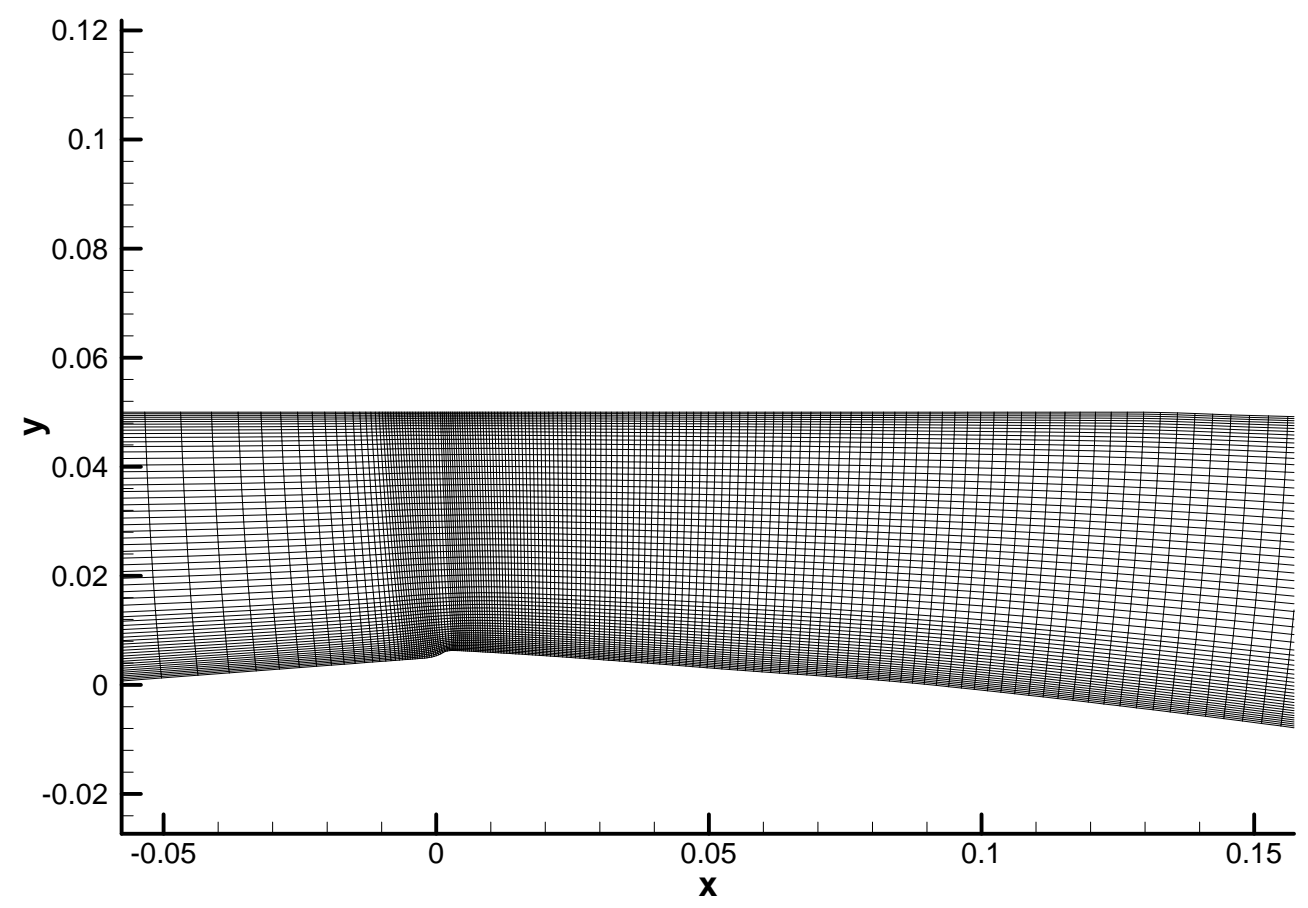

Figure 3: Enlargement of the mesh near the Venturi throat. 


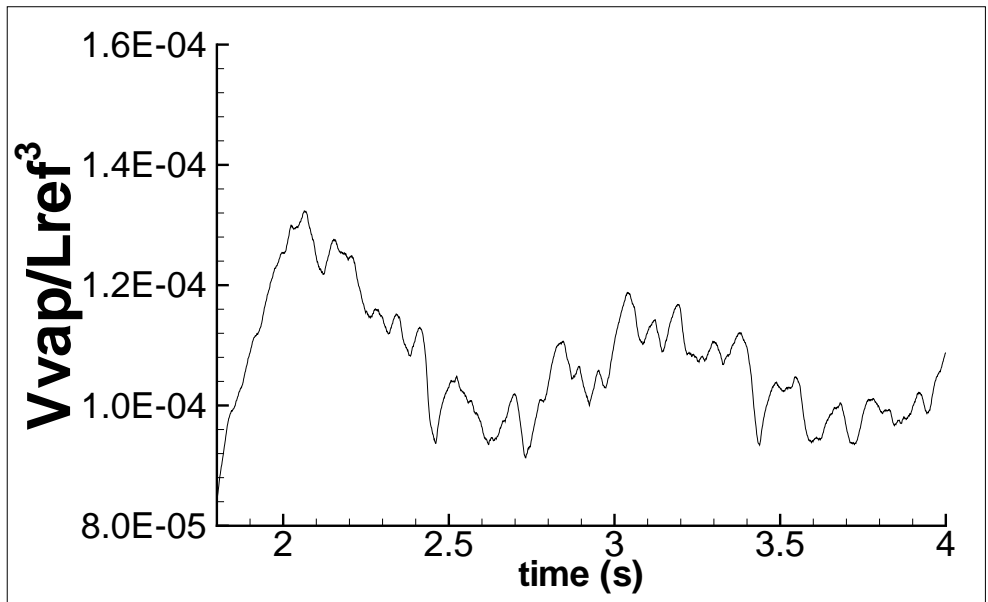

Figure 4: Dimensionless vapour volume evolution, $k-l$ model. 


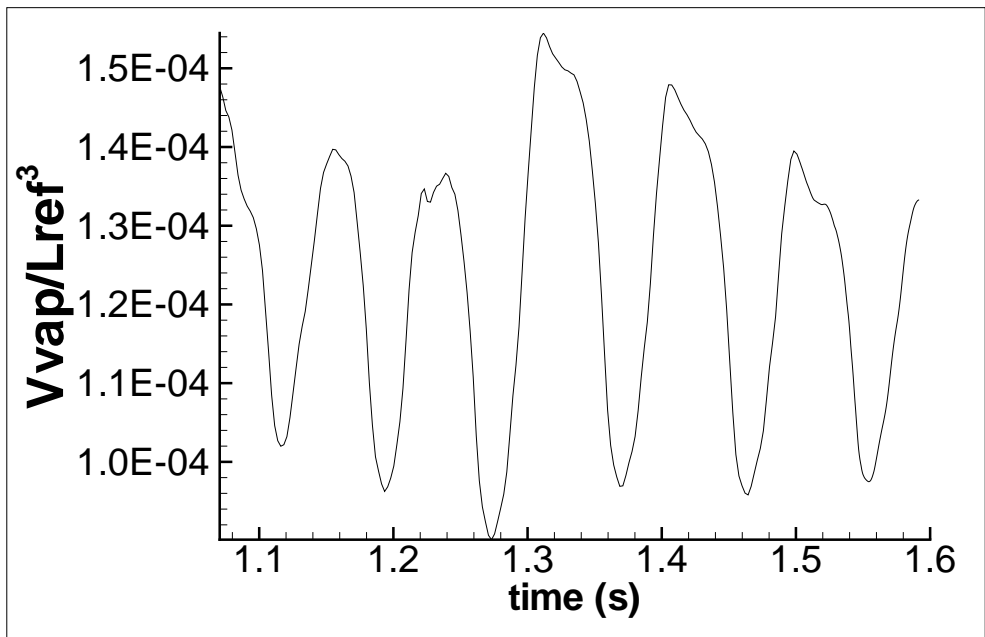

Figure 5: Dimensionless vapour volume evolution, $k-\omega$ SST model. 

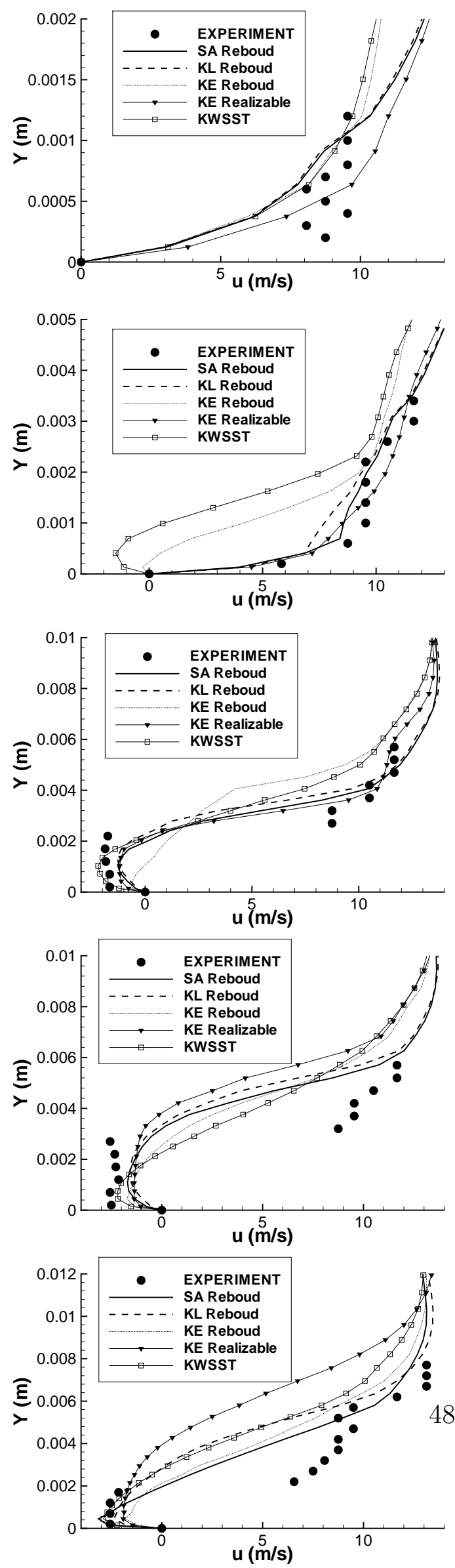

Figure 6: Velocity profiles from station 1 to 5 . 

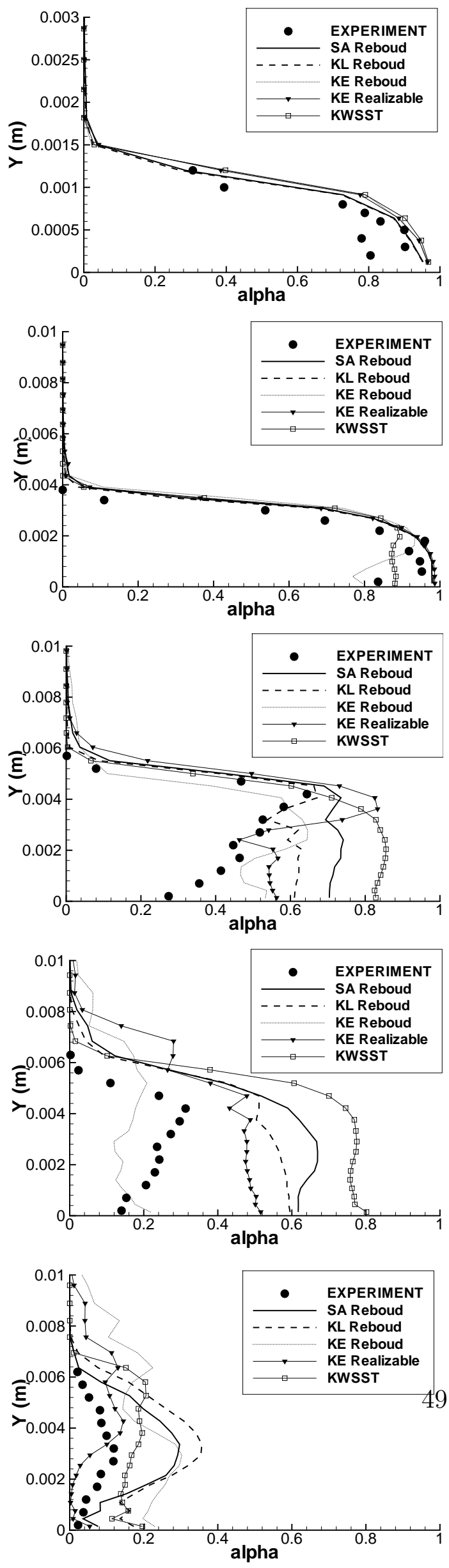

Figure 7: Void ratio profiles from station 1 to 5 . 


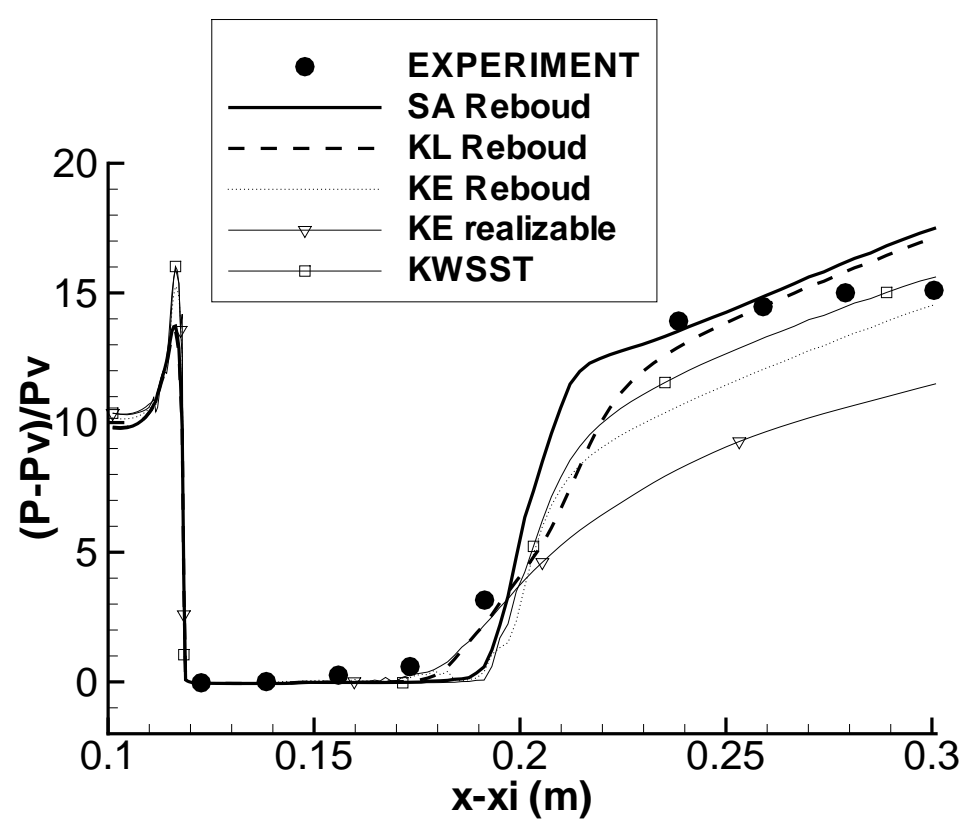

Figure 8: Dimensionless wall pressure evolution. 


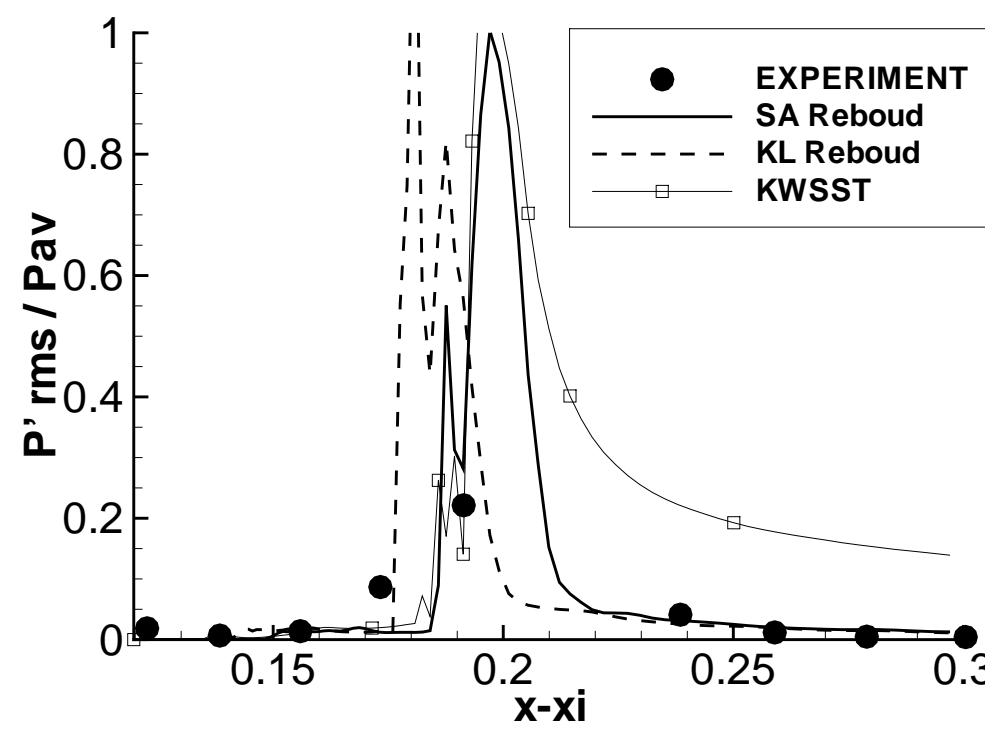

Figure 9: RMS wall pressure fluctuations. 


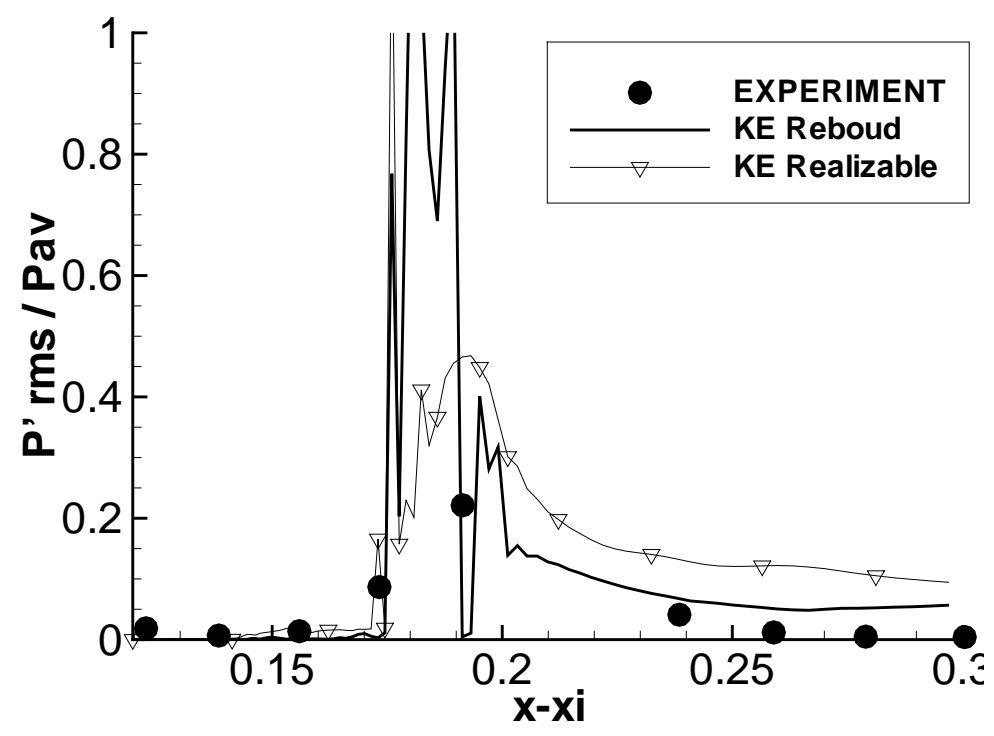

Figure 10: RMS wall pressure fluctuations, KE models. 

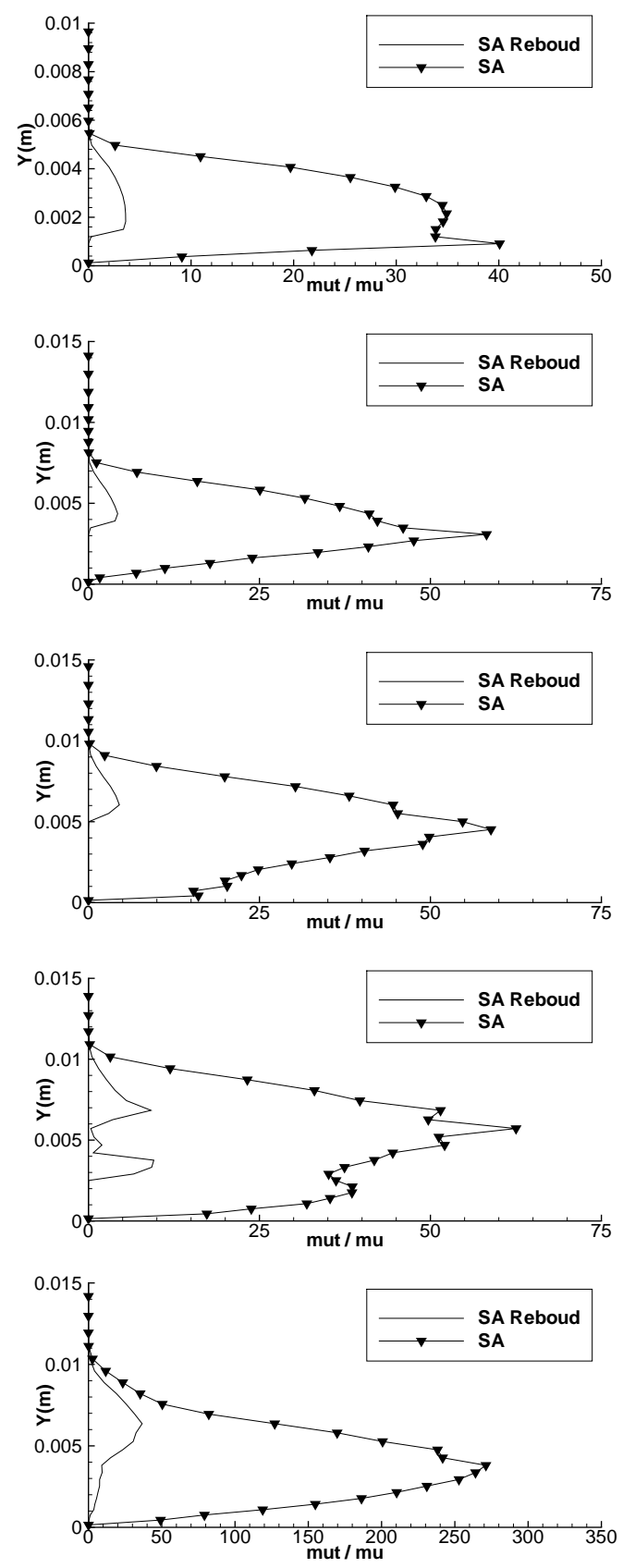

Figure 11: $\mu_{t} / \mu$ profiles from station 1 to 5 , SA models. 

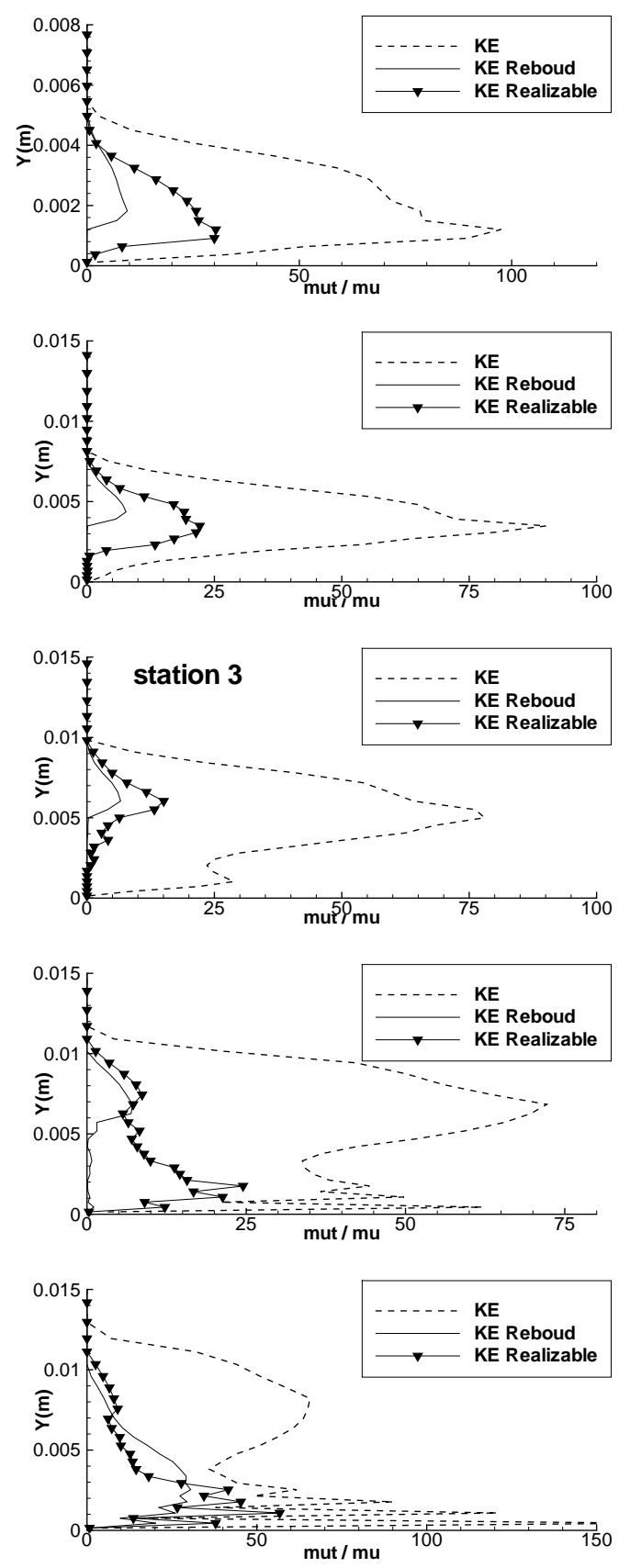

Figure 12: $\mu_{t} / \mu$ profiles from station 1 to 5 , KE models. 

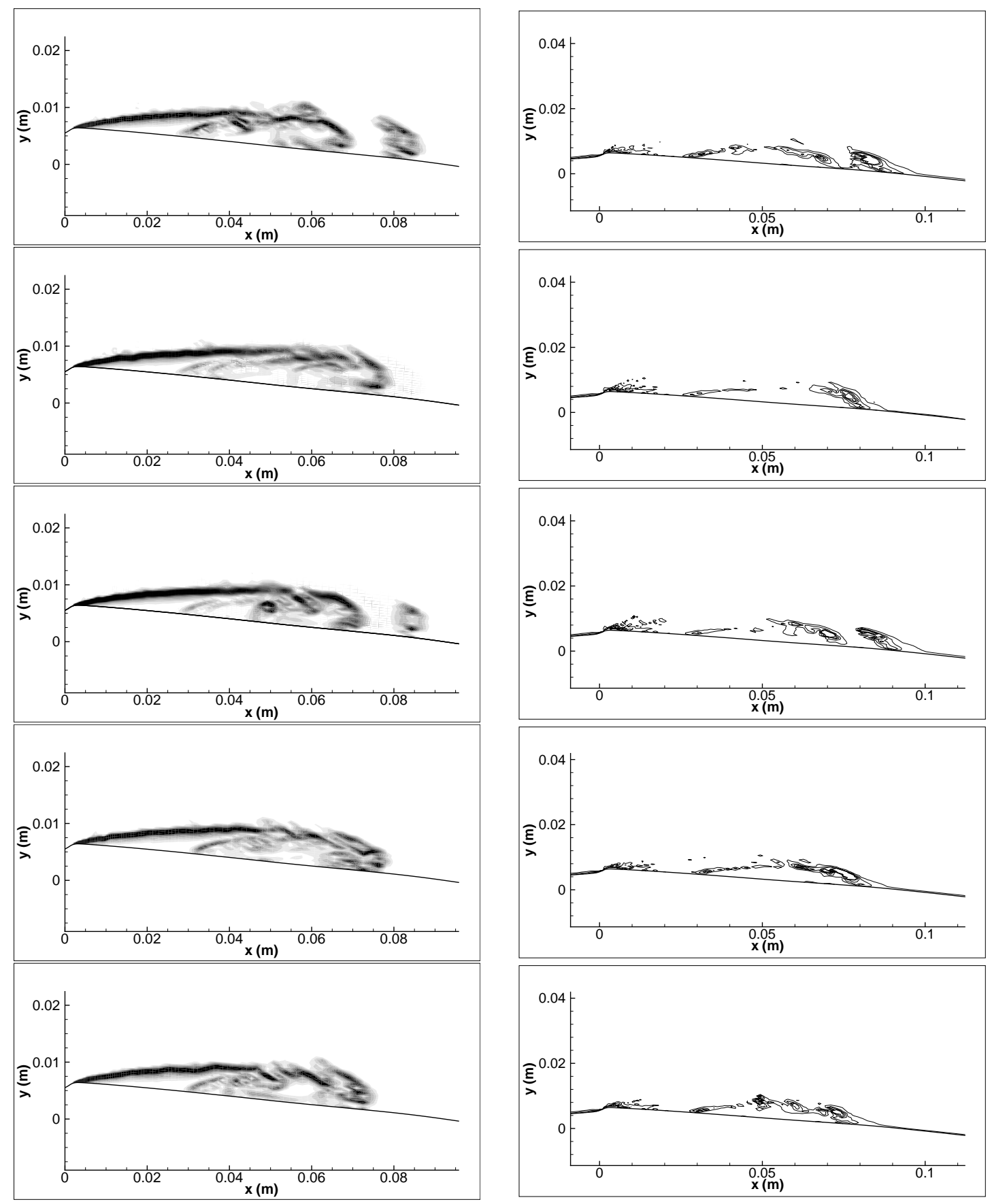

Figure 13: Contours of the density gradient (on the left) and iso-lines of the dimensionless Q-criterion (on the right), at five instants, SA Reboud model. 

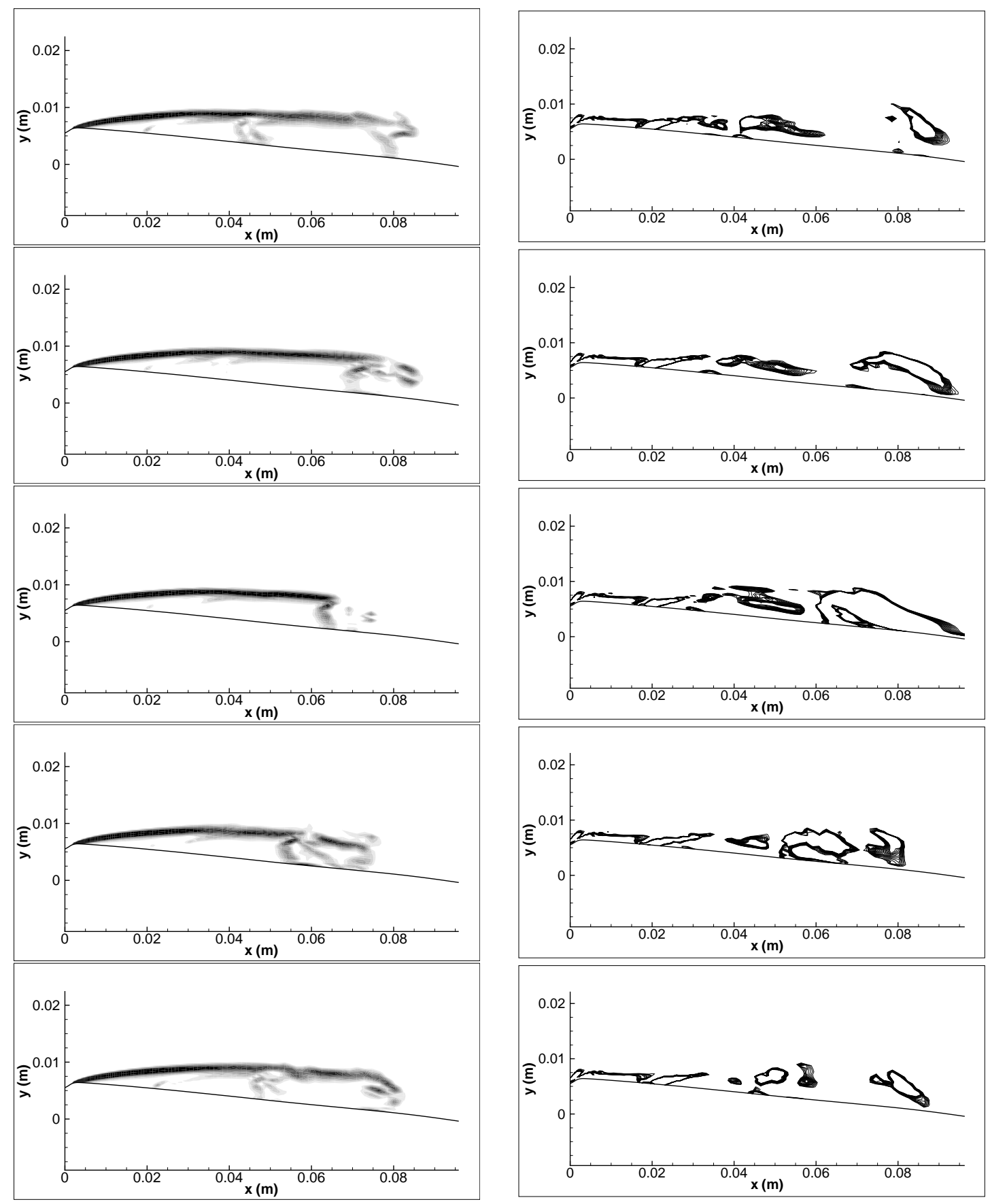

Figure 14: Contours of the density gradient (on the left) and iso-lines of the dimensionless Q-criterion (on the right), during one period, Menter SST model. 


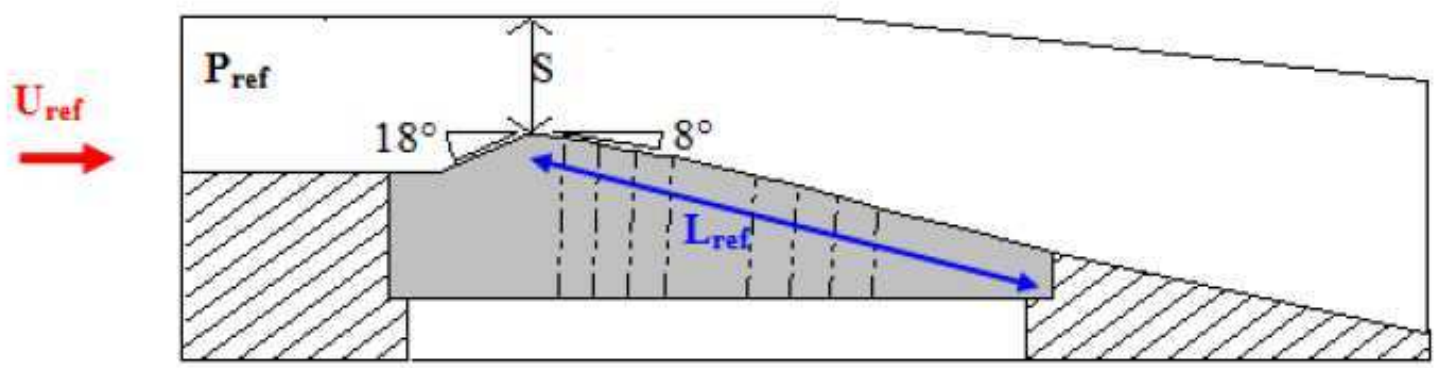

Figure 15: Schematic view of the $8^{\circ}$ Venturi profile. 


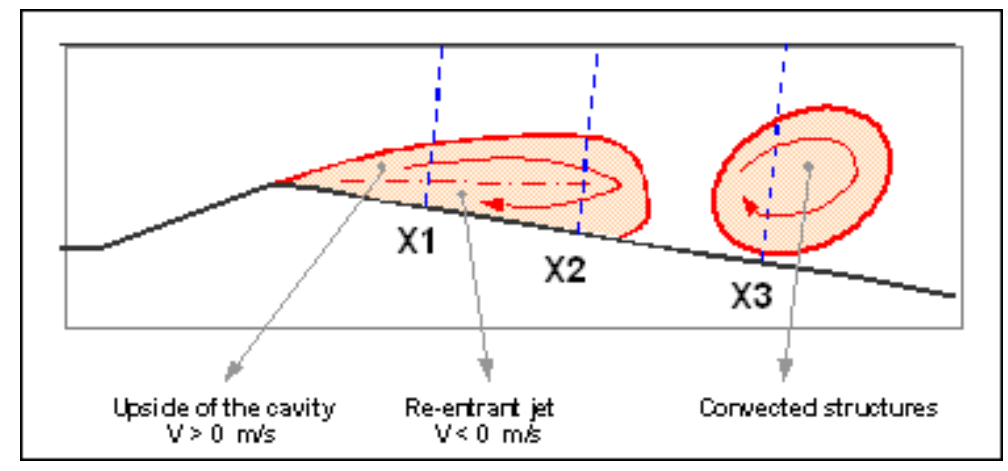

Figure 16: Schematic view of the stations and the cavity sheet. 


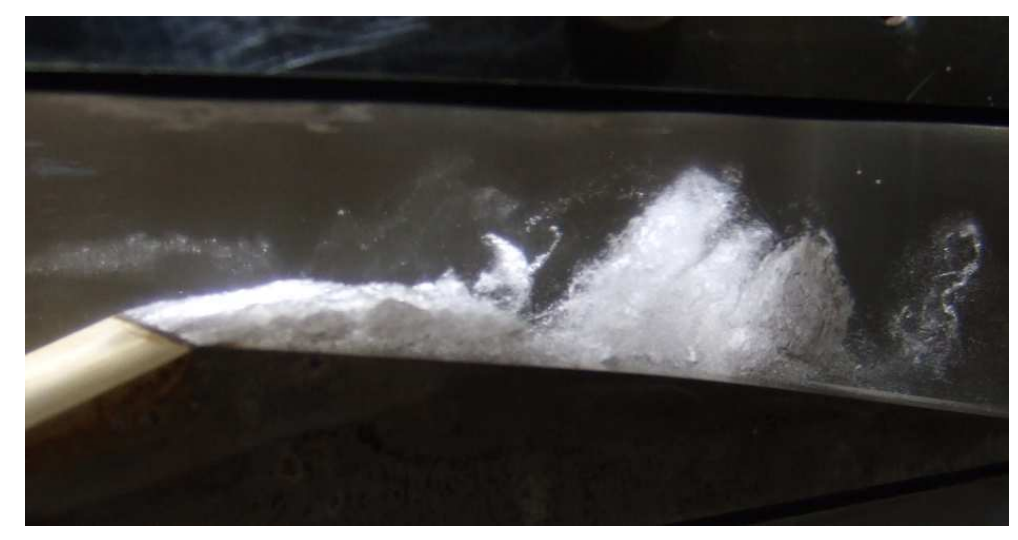

Figure 17: Photograph of the cavity. 


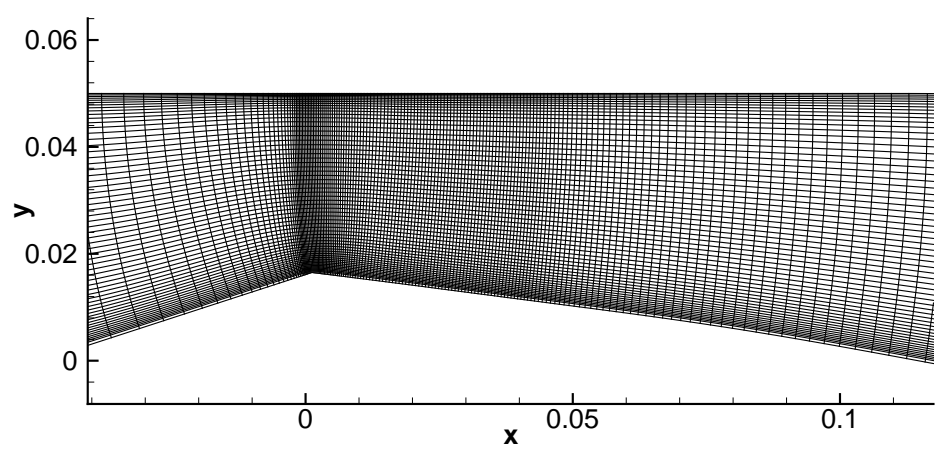

Figure 18: Enlargement of the mesh near the Venturi throat. 


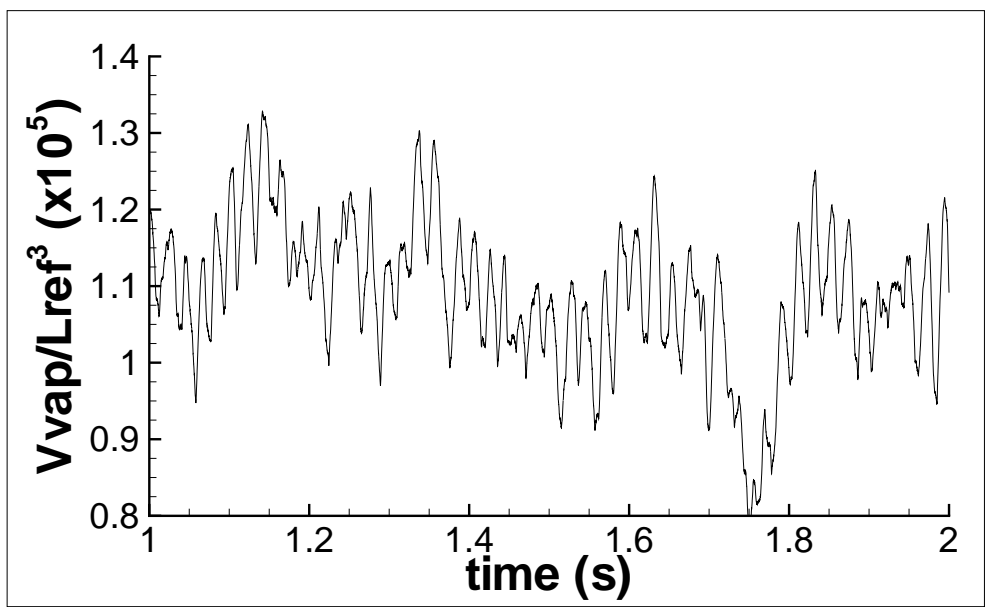

Figure 19: Dimensionless vapour volume evolution, SA Reboud model. 


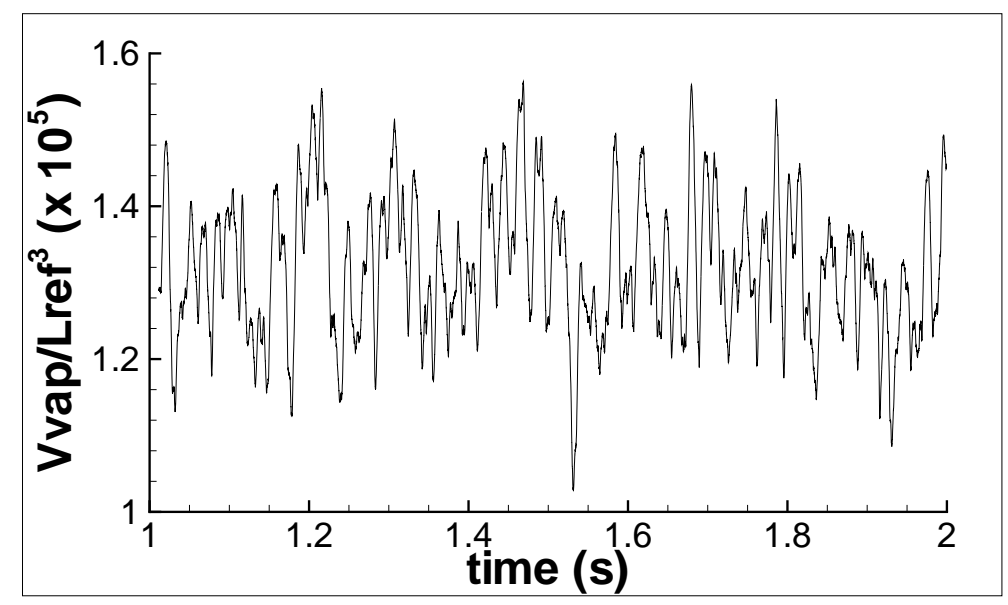

Figure 20: Dimensionless vapour volume evolution, KL Reboud model. 


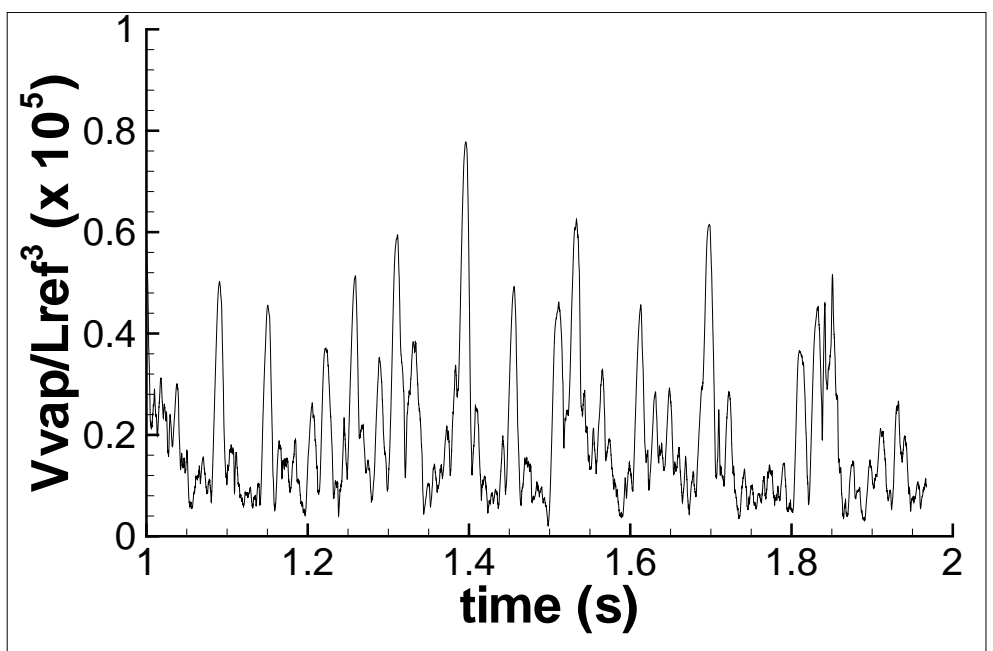

Figure 21: Dimensionless vapour volume evolution, Menter SST model. 


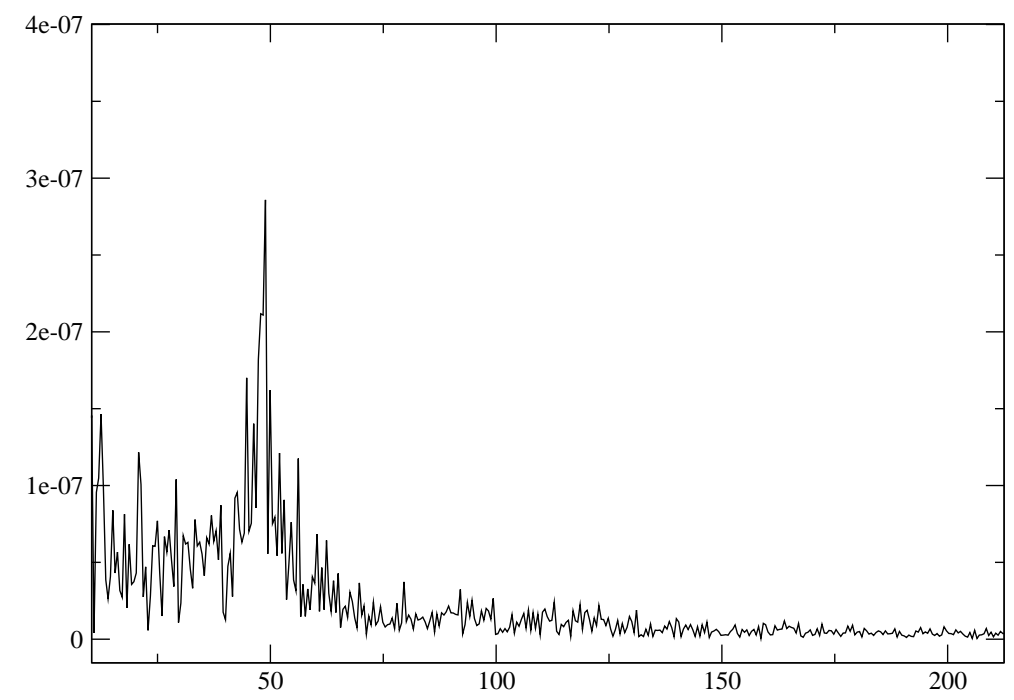

Figure 22: Vapour volume Fourier transformation, frequency (Hz), SA Reboud model. 


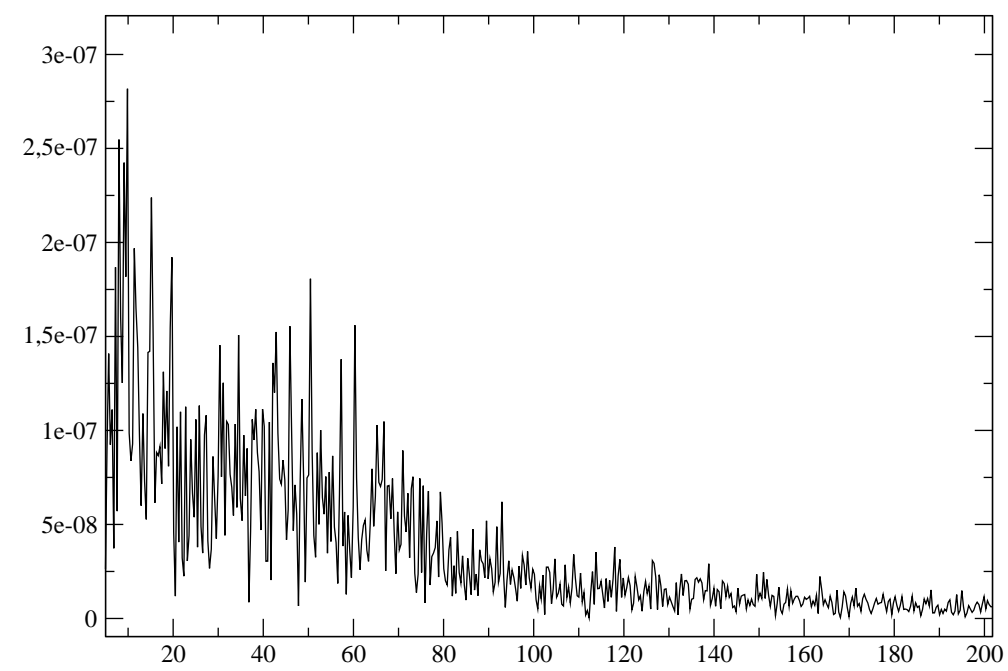

Figure 23: Vapour volume Fourier transformation, frequency (Hz), KL Reboud model. 


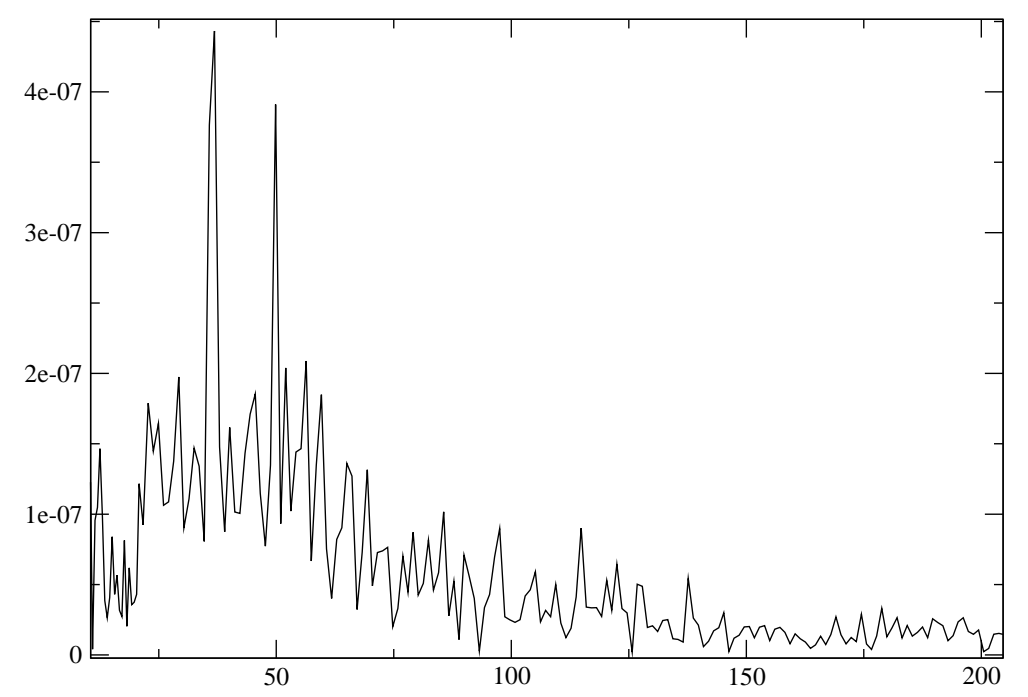

Figure 24: Vapour volume Fourier transformation, frequency $(\mathrm{Hz})$, Menter SST model. 

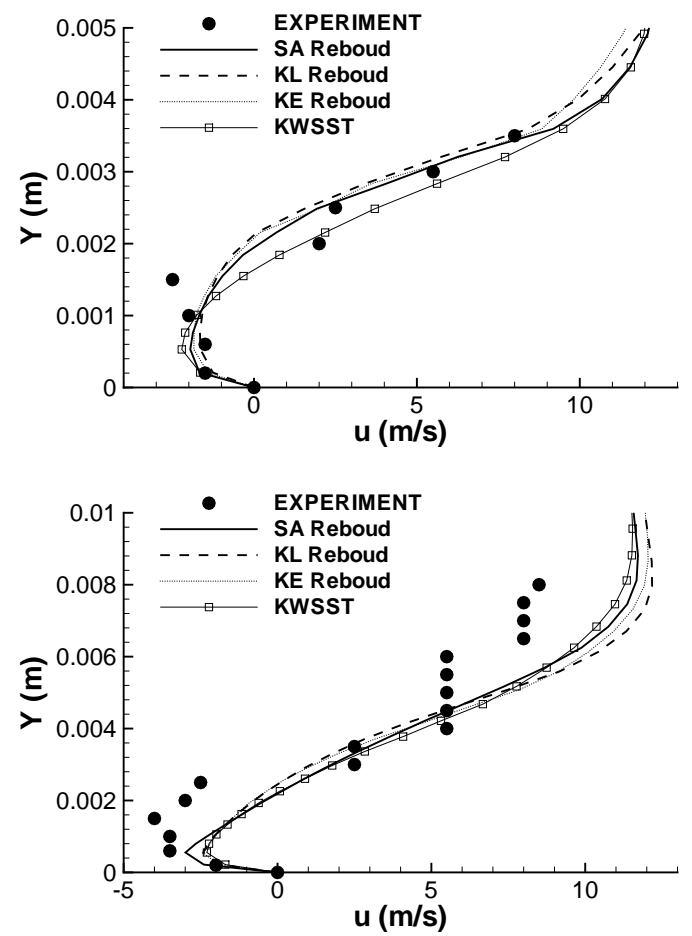

Figure 25: Velocity profiles, station 1 and 2. 

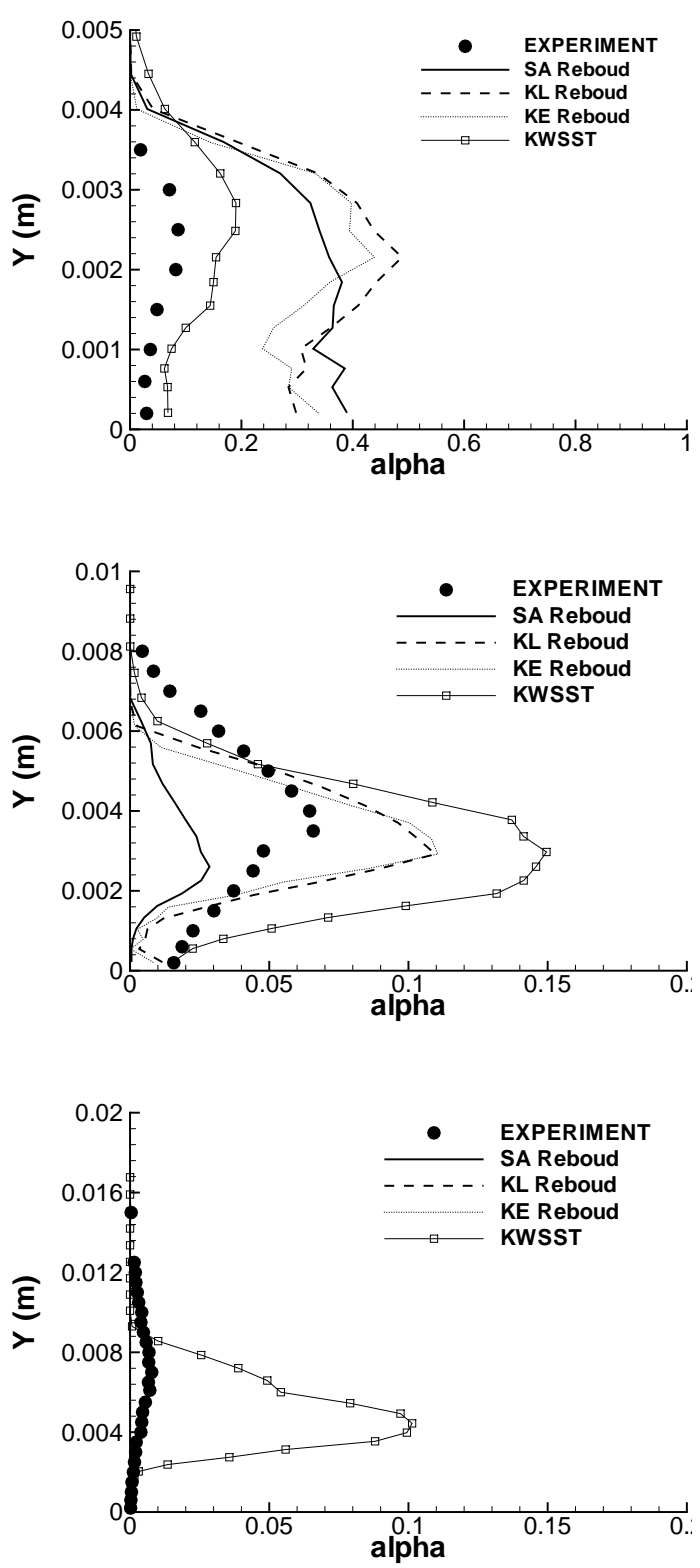

Figure 26: Void ratio profiles from station 1 to 3 . 

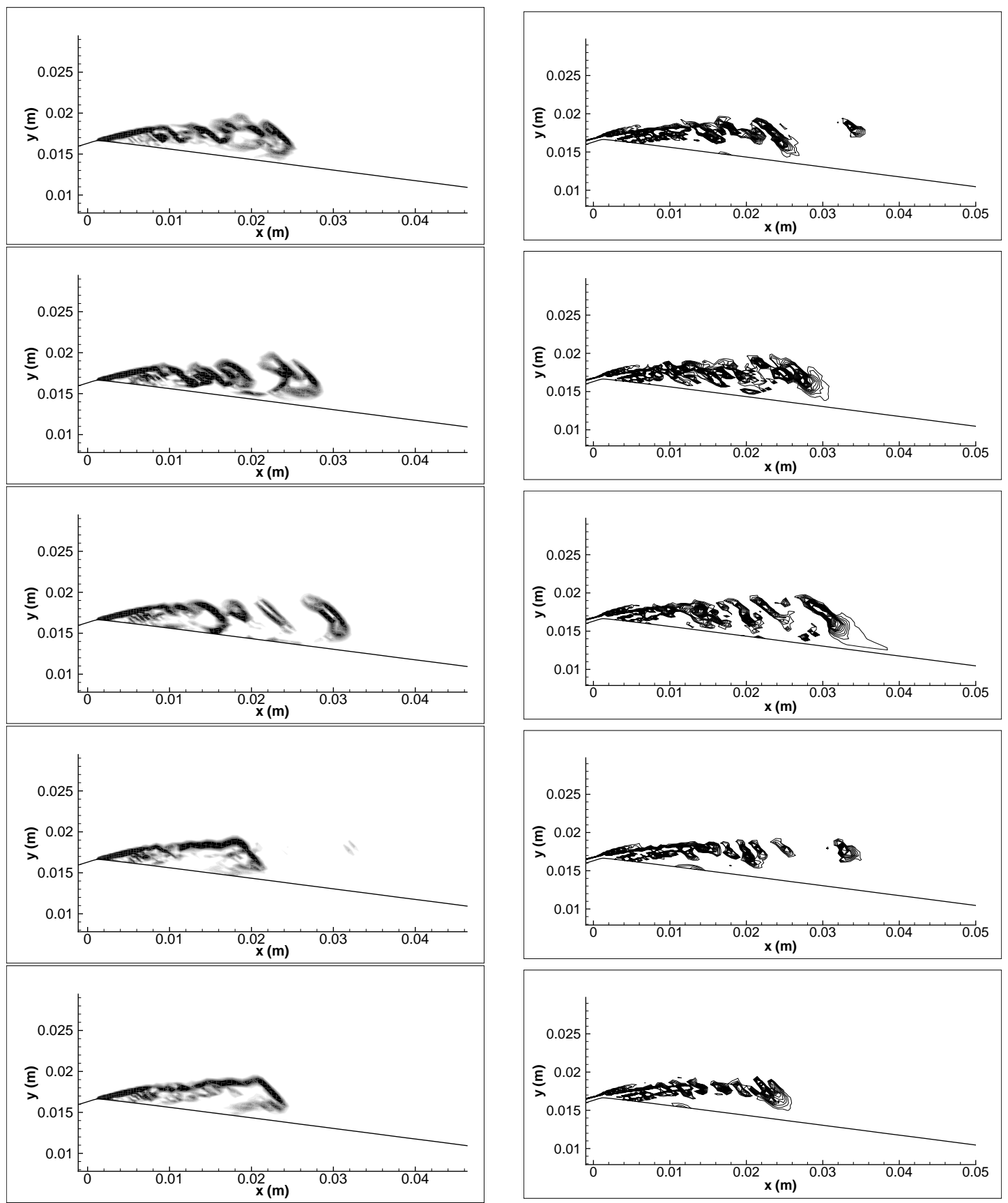

Figure 27: Contours of the density gradient (on the left) and iso-lines of the dimensionless Q-criterion (on the right) during one period, SA Reboud model. 

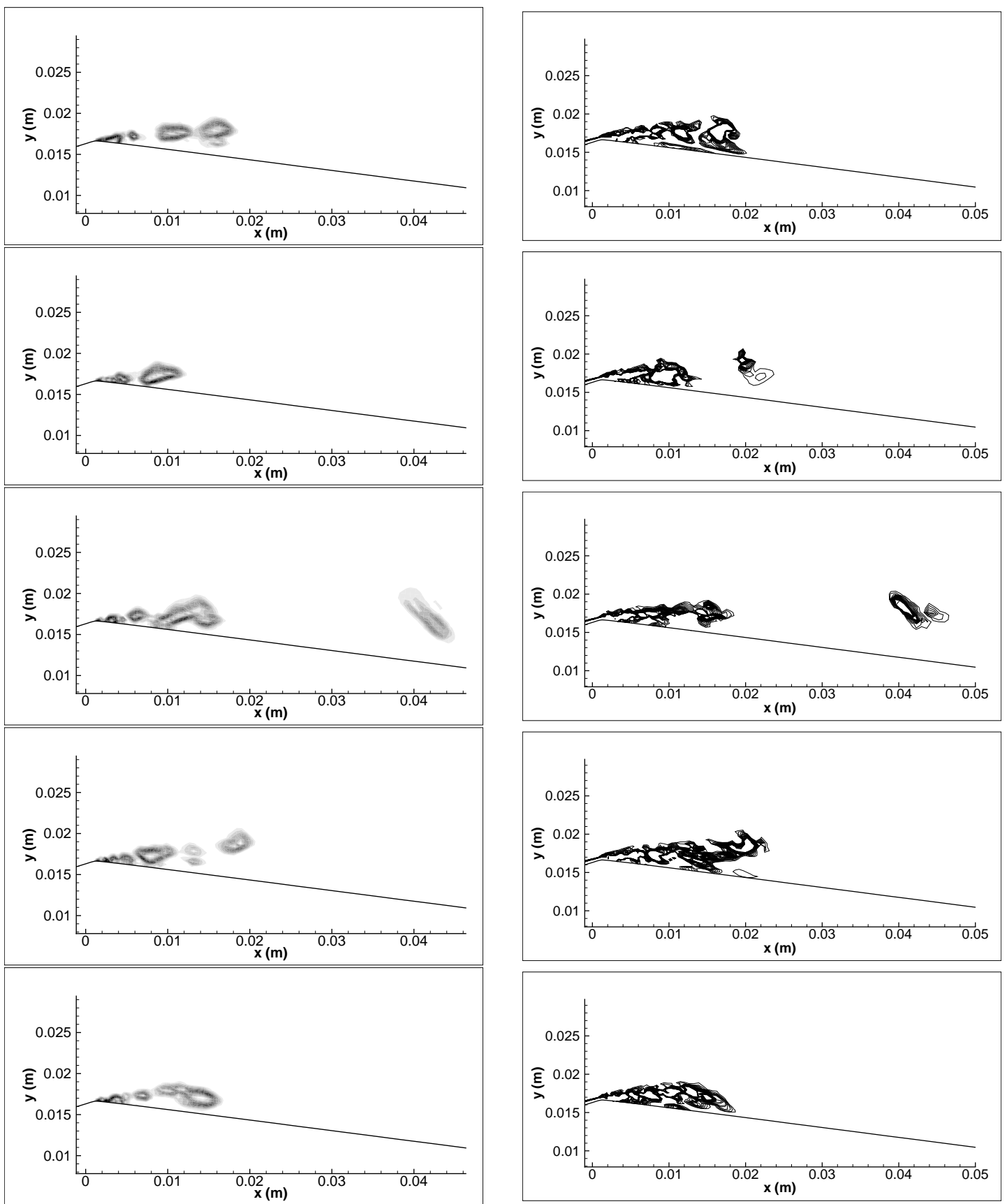

Figure 28: Contours of the density gradient (on the left) and iso-lines of the dimensionless Q-criterion (on the right) during one period, Menter SST model. 